\title{
DEEP HST/STIS VISIBLE-LIGHT IMAGING OF DEBRIS SYSTEMS AROUND SOLAR ANALOG HOSTS
}

\author{
Glenn Schneider ${ }^{1}$, Carol A. Grady ${ }^{2}$, Christopher C. Stark ${ }^{3}$, Andras Gaspar ${ }^{1}$, Joseph Carson ${ }^{4}$, \\ John H. Debes ${ }^{5}$, Thomas Henning ${ }^{6}$, Dean C. Hines ${ }^{5}$, Hannah Jang-Condell ${ }^{7}$, Marc J. Kuchner ${ }^{3}$, Marshall Perrin ${ }^{5}$, \\ Timothy J. Rodigas ${ }^{8}$, Motohide Tamura ${ }^{9}$, and John P. Wisniewski ${ }^{10}$ \\ ${ }^{1}$ Steward Observatory and the Department of Astronomy, The University of Arizona, \\ 933 North Cherry Avenue, Tucson, AZ 85721, USA; gschneider@as.arizona.edu \\ ${ }^{2}$ Eureka Scientific, 2452 Delmer, Suite 100, Oakland, CA 96002, USA \\ ${ }^{3}$ NASA/Goddard Space Flight Center, Exoplanets \& Stellar Astrophysics Laboratory, Code 667, Greenbelt, MD 20771, USA \\ ${ }^{4}$ Department of Physics and Astronomy, College of Charleston, 66 George Street, Charleston, SC 29424, USA \\ ${ }^{5}$ Space Telescope Science Institute, 3700 San Martin Drive, Baltimore, MD 21218, USA \\ ${ }^{6}$ Max-Planck-Institut für Astronomie, Königstuhl 17, D-69117, Heidelberg, Germany \\ ${ }^{7}$ Department of Physics and Astronomy, University of Wyoming, Laramie, WY 82071, USA \\ ${ }^{8}$ Department of Terrestrial Magnetism, Carnegie Institute of Washington, 5241 Branch Road, NW, Washington, DC 20015, USA \\ 9 The University of Tokyo, National Astronomical Observatory of Japan, 2-21-1 Osawa, Mitaka, Tokyo, 181-8588, Japan \\ ${ }^{10}$ H. L. Dodge Department of Physics and Astronomy, University of Oklahoma, 440 West Brooks Street, Norman, OK 73019, USA \\ Received 2016 April 27; revised 2016 May 27; accepted 2016 May 31; published 2016 August 19
}

\begin{abstract}
We present new Hubble Space Telescope observations of three a priori known starlight-scattering circumstellar debris systems (CDSs) viewed at intermediate inclinations around nearby close-solar analog stars: HD 207129, HD 202628, and HD 202917. Each of these CDSs possesses ring-like components that are more massive analogs of our solar system's Edgeworth-Kuiper Belt. These systems were chosen for follow-up observations to provide imaging with higher fidelity and better sensitivity for the sparse sample of solar-analog CDSs that range over two decades in systemic ages, with HD 202628 and HD 207129 (both 2.3 Gyr) currently the oldest CDSs imaged in visible or near-IR light. These deep (10-14 ks) observations, made with six-roll point-spread-function template visible-light coronagraphy using the Space Telescope Imaging Spectrograph, were designed to better reveal their angularly large debris rings of diffuse/low surface brightness, and for all targets probe their exo-ring environments for starlight-scattering materials that present observational challenges for current ground-based facilities and instruments. Contemporaneously also observing with a narrower occulter position, these observations additionally probe the CDS endo-ring environments that are seen to be relatively devoid of scatterers. We discuss the morphological, geometrical, and photometric properties of these CDSs also in the context of other CDSs hosted by FGK stars that we have previously imaged as a homogeneously observed ensemble. From this combined sample we report a general decay in quiescent-disk $F_{\text {disk }} / F_{\text {star }}$ optical brightness $\sim t^{-0.8}$, similar to what is seen at thermal IR wavelengths, and CDSs with a significant diversity in scattering phase asymmetries, and spatial distributions of their starlight-scattering grains.
\end{abstract}

Key words: methods: observational - planet-disk interactions - stars: individual (HD 207129, HD 202628, HD 202917) - stars: solar-type

\section{INTRODUCTION}

Circumstellar (CS) debris systems (CDSs, also known as "disks") are increasingly observed to possess ring-like components or architectures that have been predicted both from thermal infrared excesses prior to direct imaging and from theoretical models of disk dynamics. Such structures may be indicative of perturbing forces from unseen co-orbiting planets, or other influences (e.g., in the presence of remnant gas) that may sculpt the starlight-scattering materials in these systems into ring-like morphologies. High-contrast imaging of CDSs at optical and near-IR wavelengths with space-based coronagraphy, i.e., with the Hubble Space Telescope (HST), and groundbased imaging systems augmented by adaptive optics on large telescopes (e.g., GPI, SPHERE, MagAO, SCExAO, and others) have recently informed a rich diversity in CDS architectures and properties for the small number of systems currently within observational reach. This includes the occasional occurrence of starlight-scattering debris rings when observable and exo-ring material may be bound to, or escaping from, the CDSs (e.g., Schneider et al. 2014, henceforth Sch14).
This paper presents the principal observational results from a high-fidelity CDS optical imaging program using the HST Imaging Spectrograph (STIS) undertaken, in part, to study the ring-like debris systems hosted by three nearby close-solar $(\mathrm{G} 2 \mathrm{~V}-\mathrm{G} 7 \mathrm{~V})$ analog stars. Herein we focus on the observational data themselves with some relevant discussions of interpretive analysis. More in-depth modeling and further consideration of the analysis quality (AQ) scattered-light images presented within are the subjects of future papers.

In Section 1 of this paper we introduce the continuing relevance and utility of the symbiotic uniqueness space for $H S T /$ STIS scattered-light imaging of CDS materials and architectures. In Section 2 we discuss and present the target sample in the context also of other related and precursor observations. In Section 3 we discuss the observational plan and technical methodology. In Section 4 we review the need for, and our selection of, point spread function (PSF) calibration template stars. In Section 5 we review the data reduction and calibration processes and techniques applied to the observational data. In Section 6 we describe a simple morphological scattered-light model for ring-like components 
Table 1

G-star Target Sample of HST GO 13786

\begin{tabular}{|c|c|c|c|c|c|c|c|c|}
\hline \multirow{2}{*}{ Target } & \multirow{2}{*}{$V_{\mathrm{mag}}$} & \multirow{2}{*}{$B-V$} & \multirow{2}{*}{$\begin{array}{c}\text { Spectral } \\
\text { Type }\end{array}$} & \multirow{2}{*}{$\begin{array}{l}\text { Distance } \\
(\mathrm{pc})\end{array}$} & \multirow{2}{*}{$\begin{array}{l}\text { Age }^{\mathrm{a}} \\
(\mathrm{Myr})\end{array}$} & \multirow{2}{*}{$\begin{array}{c}\text { Disk }^{\mathrm{b}} \\
L_{\mathrm{IR}} / L_{\mathrm{star}}\end{array}$} & \multicolumn{2}{|c|}{ Initial $H S T$ Imaging } \\
\hline & & & & & & & Instrument & Reference \\
\hline HD 207129 & 5.58 & +0.60 & $\mathrm{G} 2 \mathrm{~V}$ & 16.0 & $2350 \pm 850$ & $1.1 \times 10^{-4}$ & ACS & Krist et al. (2010) \\
\hline HD 202628 & 6.75 & +0.64 & G5V & 24.4 & $2300 \pm 1000$ & $1.4 \times 10^{-4}$ & ACS & Krist et al. (2012) \\
\hline HD 202917 & 8.67 & +0.65 & G7V & 43.0 & $30(+10,-20)$ & $2.9 \times 10^{-4}$ & NICMOS & Soummer et al. (2014) \\
\hline
\end{tabular}

Notes.

a Age estimations-HD 202917: Moór et al. (2006); HD 207129: Mamajek \& Hillenbrand (2008), Soderblom (1985), HD 202628: Krist et al. (2012).

${ }^{\mathrm{b}} L_{\mathrm{IR}} / L_{\mathrm{star}}$ estimations-HD 202917: Moór et al. (2006); HD 207129: Marshall et al. (2011), HD 202628: Koerner et al. (2010).

of CDSs that we have employed to estimate their primary characterizing geometrical and physical parameters. In Section 7 we discuss in detail the individual objects: HD 207129, HD 202628, and HD 202917. In Section 8 we discuss and intercompare the observationally derived results as a target class in the context of, contributing to, and expanding the prior sparse sample of imaged solar-analog CDSs. In Section 9 we provide some closing commentary.

\section{THE $H S T /$ GO 13786 TARGET SAMPLE}

In 2010-2011, in HST/GO program $12228^{11}$, we observed an $H S T$-selected sample of 11 CDSs comprising 10 debris disks and one mature protoplanetray disk. These CDSs sampled a diverse set of main-sequence host stars ranging from $\mathrm{M} 1 \mathrm{~V}-\mathrm{A} 5 \mathrm{~V}$ in spectral type and $\sim 10-1000 \mathrm{Myr}$ in age; see Sch14 and in context of Choquet et al. (2016) (see their Figure 1). These were high-fidelity follow-ups to prior discovery observations from HST's Near Infrared Camera and Multi-Object Spectrometer (NICMOS) and Advanced Camera for Surveys (ACS) instruments, homogeneously obtained using HST's Imaging Spectrograph (STIS) with six-roll PSF-template-subtracted coronagraphy (6R-PSFTSC) for all targets. This sample included a number of CDSs of intermediate to high inclination from which ring-like debris components were directly observed. This included HD 181327, HD 61005, HD 15115, HD 92945, and the close-solar (G2V) analog HD 107146. ${ }^{12}$ Most of these, as well as the nearly edge-on HD 32297, were also shown to possess extensive systems of exo-ring materials extending well beyond their bright ring radii, posited as possibly commonly occurring in CDSs. With this initial sample in hand, in follow-on studies with HST GO program 13786 we sought to extend in number and "fill-in" in age and spectral type this observationally homogeneous, but small, sample with a focused target list of five nearby CDSs of intermediate inclination, each with a priori established ring-like morphologies.

With sufficient spatial resolution and contrast ${ }^{13}$ in scatteredlight imaging, many CDSs are directly seen to possess ring-like architectures or components that may indicate the presence of planets. These are best revealed with spatially resolved imaging in systems with low to intermediate inclinations from the line of sight, i.e., close to "face on." Although such low-inclination

\footnotetext{
11 http://www.stsci.edu/cgi-bin/get-proposal-info?

$\mathrm{id}=12228$ \&observatory $=$ HST

${ }^{12}$ Herein, in Section 8.2.1, we also call attention also to the directly observed ring-like component in HD 15745.

${ }^{13}$ In this paper, image contrast is defined as the ratio between the flux density in a resel (resolution element) at any stellocentric location after coronagraphic and/or augmented starlight suppression by any other means including PSF subtraction, compared to the flux density in the central resel of an unocculted stellar PSF.
}

systems provide revealing views of the radial distribution of starlight-scattering material (e.g., Debes et al. 2016), they leave interpretive analysis of particle properties augmented through scattering phase functions ill-constrained ${ }^{14}$ (Stark et al. 2015; Hedman \& Stark 2015). Additionally, scattered-light images of low-inclination CDSs, while enabling best spatially resolved 2D mapping of the in-plane scattering material (e.g., HD 181327; Stark et al. 2014), provide limited information on the vertical structure in such systems. Conversely, azimuthal structure in edge-on (high-inclination) disks such as $\beta$ Pic (Apai et al. 2015) and AU Mic (Sch14, Boccaletti et al. 2015) is self-obscured by the viewing geometry, though with better views of the vertical (out of disk-plane) material in the system. While high-inclination CDSs provide nearly a full range of scattering phase angles, the tomographic view of the scattering particles is conflated by the line-of-sight-integrated viewing geometry through the plane of the disk. Therefore, intermediate-inclination CDSs can provide a best "compromise" in viewing geometries to simultaneously explore the in-plane and vertical distribution of starlight-scattering materials and provide largely unobstructed views of the system architectures. Many of these CDS structures currently remain best revealed with $H S T$ coronagraphy symbiotic to capabilities provided by suitably instrumented large ground-based telescopes with high-order adaptive-optics systems.

Our GO 13786 sample included two target classes that a priori were sampled only sparsely (with direct imaging): first, and the subject of this paper, a G-star sample of three closesolar analog stars both younger $(\lesssim 80 \mathrm{Myr})$ and older $(\gtrsim 2 \mathrm{Gyr})$ than those imaged prior in $H S T / G O 12228$. The principal characteristics of these targets are summarized in Table 1. Second, a "revisit" to better explore both the innermost and the outermost light-scattering regions of two iconic and youthful ( 5-8 Myr) higher-stellar-mass (spectral types A0V, B9V) debris systems: HR 4796A and HD 141569A-both observationally challenging owing to the presence of M-star companions. These are the subjects of two companion papers: G. Schneider et al. (2016, in preparation) and Konishi et al. (2016), as discussed further therein.

\section{OBSERVATIONS}

We obtained STIS coronagraphic observations of our three G-star CDS targets, along with their contemporaneously interleaved PSF-template stars, as part of $H S T / \mathrm{GO}$ program $13786^{15}$ (G. Schneider: PI). All observations were made during

\footnotetext{
14 Explorable range of scattering phase angle: $\varphi=\cos ^{-1}(\sin i \cos \theta)$; $i=$ inclination, $\theta=$ azimuth angle in disk plane.

15 http://www.stsci.edu/cgi-bin/get-proposal-info? $\mathrm{id}=13786 \&$ observatory $=$ HST
} 
Table 2

Observation and Data Log for Solar Analog Targets and PSF Template Stars in HST GO 13786

\begin{tabular}{|c|c|c|c|c|c|c|c|}
\hline $\begin{array}{l}\text { Target } \\
\text { (Disk/PSF) }\end{array}$ & $\begin{array}{l}\text { UT Date } \\
\text { Obs. Start }\end{array}$ & $\begin{array}{l}\text { Orient } \\
\text { (deg) }\end{array}$ & $\begin{array}{l}\text { WA0.6 } \\
\text { \# Exp }\end{array}$ & $\begin{array}{l}\text { WA0.6 } T_{\text {EXP }} \\
\text { all visits (s) }\end{array}$ & $\begin{array}{l}\text { WA1.0 } \\
\text { \# Exp }\end{array}$ & $\begin{array}{l}\text { WA1.0 } T_{\mathrm{EXP}} \\
\text { all visits }(\mathrm{s})\end{array}$ & $\begin{array}{c}\text { Visit } \\
\text { Data ID }\end{array}$ \\
\hline HD 202917 & 2015 Oct 13 & $36.1,14.1,352.1$ & 24 & 2400.0 & 9 & 4437.0 & $51,52,54$ \\
\hline LTT 8893 & 2015 Oct 13 & 2.6 & 10 & 264.0 & 13 & 1677.0 & 53 \\
\hline HD 202917 & 2015 Aug 01 & $321.1,300.6,280.1$ & 24 & 2400.0 & 9 & 4797.0 & $55,56,58$ \\
\hline LTT 8893 & 2015 Aug 01 & 285.1 & 10 & 264.0 & 4 & 2114.4 & 57 \\
\hline HD 207129 & 2015 May 26 & $236.7,214.2,191.7$ & 72 & 417.6 & 39 & 4524.0 & $15,16,18$ \\
\hline $\mathrm{Tau}^{1} \mathrm{Gru}$ & 2015 May 26 & 203.1 & 23 & 204.7 & 8 & 1427.2 & 17 \\
\hline Tau $^{1}$ Gru-cal $^{\mathrm{b}}$ & 2015 May 26 & 203.1 & $\cdots$ & $\cdots$ & 2 & 178.4 & 17 \\
\hline HD 207129 & 2015 Aug 08 & $311.7,289.7,267.7$ & 72 & 417.6 & 39 & 4524.0 & $11,12,14$ \\
\hline $\mathrm{Tau}^{1} \mathrm{Gru}$ & 2015 Aug 08 & 268.3 & 23 & 204.7 & 8 & 1427.2 & 13 \\
\hline $\mathrm{Tau}^{1} \mathrm{Gru}^{-\mathrm{cal}^{\mathrm{b}}}$ & 2015 Aug 08 & 268.3 & $\ldots$ & $\ldots$ & 2 & 178.4 & 13 \\
\hline HD 202628 & 2015 Sep 17 & $341.1,1.6,21.1$ & 60 & 1032.0 & 15 & 4890 & $01,02,04$ \\
\hline HR 8042 & 2015 Sep 17 & 6.5 & 20 & 310.0 & 6 & 1320 & 03 \\
\hline HD 202628 & 2015 May 30 & $243.1,220.1,197.1$ & 60 & 1032.0 & 15 & 4890 & $05,06,08$ \\
\hline $\mathrm{BX} \mathrm{Mic}^{\mathrm{c}}$ & 2015 May 30 & 215.1 & 20 & 366.0 & 5 & 1570 & 07 \\
\hline
\end{tabular}

Notes.

a (Single-orbit) visit-level data set ID as assigned by MAST. GO 13786 data archived as ocjc + datset_id $+*$.

${ }^{\mathrm{b}}$ Same target-Tau ${ }^{1} \mathrm{Gru}$ : calibration and check exposures-not used directly for creation of PSF subtraction template.

${ }^{c}$ V07: background star in close angular proximity and/or companion revealed-ill-suited as PSF subtraction template.

the interval 2015 May-October. For all targets we closely followed the successful observational paradigm for $H S T$ multiroll PSF-template subtraction coronagraphy as previously demonstrated in HST GO program 12228 and detailed by Sch14. For each G-star CDS and its paired PSF-template star, two sets of four contiguous single-orbit visits were programed for coronagraphic observations using STIS occulting wedge A. In each orbit, Wedge-A was used at both its 0". 6 (WA0.6) and 1!"0 (WA1.0) wide locations, in combination with exposure times used (see Table 2), for high-dynamic-range imaging (with instrumental GAIN $=4$ ) over stellocentric angles ranging from 0 ". 3 to $>10^{\prime \prime}$. Each CDS target was observed at six spacecraft roll angles in two sets of three orbits separated by 2-4 months due to HST differential roll constraints at any epoch. ${ }^{16}$ At each epoch the PSF-template star for each CDS target was similarly observed in a single orbit interleaved, contiguously, between the second and third CDS orbits (thus a total of eight orbits required for each CDS target and its PSFtemplate star). Within each orbit, the target (CDS-host or PSFtemplate star) was first placed at the WA0.6 position, imaged for roughly $1 / 3$ of an orbit multiple times to a nearly full-well depth where brightest (not to exceed image saturation at the edges of the occulting wedge), then repositioned at WA1.0 and more deeply imaged multiple times for the remainder of the orbit.

Table 2 gives a summary of the observations, with further details available through the STScI MAST archive. The rationale for the details of the observational design is given in Sch14. Columns 1 and 2, respectively, give the names of the disk-host and corresponding interleaved PSF-template stars, and the UTC date of the start of each of the four-orbit contiguous observing sequences. Column 3 gives the absolute orientation angle of the images (FITS header keyword

\footnotetext{
16 The absolute celestial orientation angles for all G-star visits were unconstrained in scheduling the observations, but the relative orientations (differential between visits) for the CDS targets were optimally (ideally) constrained for sequential visit-to-visit orientation differentials of $\sim 30^{\circ}$. This, however, could not always be closely realized due to restrictions on $H S T$ guide stars and other pointing restrictions; see Table 2 for details of the as-observed "orient" angles.
}

ORIENTAT) measured from the image $+Y$ axis to celestial north counterclockwise through east about the target star. Column 4 indicates the total number of individual WA0.6 exposures for that epoch, equally divided with multiple visits. For example, for the first entry in the table (HD 202917) it is 3 visits $\times 8$ exposures per visit $=24$ exposures. Column 5 gives the total exposure time for all corresponding WA0.6 exposures in the visit set. For example, 24 exposures $\times 100 \mathrm{~s}$ per exposure $=2400 \mathrm{~s}$. Columns 6 and 7 are similar to Columns 4 and 5, but for WA1.0 images. Column 8 gives the visit number (ID) as used in the MAST identification of data sets unique to GO program 13786 (ocjc + data ID).

\section{PSF-TEMPLATE STARS}

Beyond raw coronagraphy, the remaining incompletely suppressed starlight in the PSF halo polluting a CDS image is further reduced by subtracting a PSF template derived from a diskless star observed in a nearly identical manner. PSFtemplate stars of at least roughly comparable brightness to paired CDS stellar host targets were selected and scheduled with temporal, roll-range, and pointing constraints, to minimize otherwise systematically enhanced PSF-subtraction residuals. Template stars were selected and observations were planned with respect to their paired CDS targets to simultaneously meet the following criteria as closely as possible: (a) identically matched in $B-V$ color $(\Delta[B-V]=0.0)$, (b) change in telescope attitude (slew distance) from target to template star and back to target of $<10^{\circ}$, (c) change in telescope roll angle $<20^{\circ}$ from the middle of the three target star visits to the template star visit, (d) contiguous four-orbit visit sets with interleaved PSF visit as third with no interruptions other than Earth occultations. Details of the PSF star selection and scheduling are given in Table 3.

\section{DATA CALIBRATION AND REDUCTION}

Instrumental calibration and reduction. In processing the raw coronagraphic images into fully reduced AQ images for metrical and interpretive analysis, we follow the methodology 
Table 3

Planned, Contemporaneously Interleaved, PSF-Template Stars

\begin{tabular}{|c|c|c|c|c|c|c|c|c|}
\hline \multirow{2}{*}{$\begin{array}{l}\text { Disk } \\
\text { Target }\end{array}$} & \multirow{2}{*}{$\begin{array}{l}\text { PSF } \\
\text { Star }\end{array}$} & \multirow{2}{*}{$\begin{array}{c}\text { PSF } \\
\text { Spectral Type }\end{array}$} & \multirow{2}{*}{$\begin{array}{c}\text { PSF } \\
V\end{array}$} & \multirow{2}{*}{$\begin{array}{c}\text { PSF } \\
B-V\end{array}$} & \multirow{2}{*}{$\Delta(B V)^{\mathrm{a}}$} & \multirow{2}{*}{$\begin{array}{l}\text { PSF } \\
\text { Slew }^{b}\end{array}$} & \multicolumn{2}{|c|}{ PSF $\triangle \mathrm{ONR}$} \\
\hline & & & & & & & $\mathrm{c}$ & \\
\hline HD 207129 & Tau1 Gru & G0V & 6.04 & +0.62 & -0.03 & 11.0 & +11.1 & +21.4 \\
\hline HD 202628 & $\mathrm{BX} \mathrm{Mic}$ & G0IV & 6.82 & +0.64 & 0.00 & $10^{\circ} 4$ & $-25^{\circ} .4$ & $\ldots$ \\
\hline & HR 8042 & G3IV & 6.64 & +0.68 & -0.04 & 2.3 & $\ldots$ & $+5^{\circ} 0$ \\
\hline HD 202917 & LTT 8893 & G3V & 7.22 & +0.66 & -0.01 & 7.7 & +11.5 & +15.5 \\
\hline
\end{tabular}

Notes.

${ }^{a}$ Difference in $B-V$ color index between target and PSF-template stars; $\Delta(V R)$ used as a secondary ranking criterion.

b Angular distance in the sky between the disk target and PSF template star.

" ONR: "Off nominal roll"; $\triangle \mathrm{ONR}$ : change in roll angle between second target visit and PSF star visit in contemporaneous set.

and techniques discussed by Sch14, to which the reader is referred for details. In summary: (1) We instrumentally calibrate the individual raw images to produce 'FLT' images corrected for bias, dark current, non-linearity, and flat-field artifacts using the calstis $\mathrm{S} / \mathrm{W}$ with calibration reference files provided by STScI (CDBS system) updated to the epoch of the individual observations. (2) The location of the occulted target star in each FLT image (and its uncertainty by dispersion) is determined using the "X marks the spot method," described by Sch14 (Section 5.4 therein). (3) For each visit, the multiple FLT images obtained at the location of each occulting wedge were vetted for anomalies (e.g., image "drift"; none found within typical two-star fine-lock intra-orbit pointing stability) and then median-combined (for cosmic-ray elimination and building signal-to-noise ratio $(\mathrm{S} / \mathrm{N})$ ) into visit-level count-rate images. (4) For each visit, the visit-level WA0.6 and WA1.0 PSF template images were subtracted from the corresponding, step 3, target images. Subtraction is accomplished as in Sch14 by treating the intensity of the PSF template image (brightness in instrumental counts s ${ }^{-1}$ pixel $^{-1}$ ) and star location imaged on the detector as free parameters. Differences are simultaneously minimized in iterative subtraction in regions along the HST diffraction spikes unaffected by the STIS occulting masks and where not dominated by disk flux in target images as explained in Sch14. (5) Visit-level PSF-subtracted images (in the instrument orientation frame) are rotated to a common celestial orientation (north "up") about the occulted star and mediancombined, masking the STIS occulting wedges, diffraction spike residuals, regions of image saturation near the wedge edges, and any image anomalies in individual images. (6) Chromatic residual correction (derived from individual visits) is applied, if needed, for imperfect PSF-template colormatching as explained in Sch14 (see Section 5.6 and, e.g., Figure 17 therein). (7) The W0.6A and W1.0A images are combined with selective masking for the separate regions unsampled or degraded in one but not the other, producing a "final" (single) AQ data image over the largest stellocentric and azimuth-angle ranges afforded by both occulting wedge locations and a very large (target-dependent) imaging dynamic range in surface brightness.

Absolute photometric calibration. In this paper we report results of photometric observations in both instrumental countrate units of counts $\mathrm{s}^{-1}$ pixel $^{-1}$ (with 1 count $=4.016$ photoelectrons for "GAIN = 4") and in physical units in flux density (Jy) or surface brightness (Jy $\operatorname{arcsec}^{-2}$ ). For the latter, we adopt the STIS instrumental calibration as codified in the HST synthetic photometry package SYNPHOT (calcphot task) with
1 count $\mathrm{s}^{-1}$ pixel $^{-1}($ with GAIN $=4)=4.55 \times 10^{-7} \mathrm{Jy}$, and an absolute photometric zero-point calibration such that a star of magnitude 0 (Vega system) produces a total flux density of 2923 Jy in the STIS (unfiltered) 50CCD spectral bandpass. In assessing the brightness of observed CS debris at visible wavelengths, we assume that reflectance by the starlightscattering material is spectrally neutral (i.e., the debris is the same "color" as the host star). This may not be correct, but no filter-band (or spectro-)photometry necessary to assess a "color correction" under the broad STIS 50CCD bandpass is available (or currently possible), but an assumption of near spectral flatness should be a close approximation. For absolute calibration of surface brightness (SB; i.e., flux density per unit area) we adopt the a priori determined image scale of the STIS 50CCD channel as $0.05077 \operatorname{arcsec~pixel}^{-1}$ on both squarepixel axes. For SB, this gives 1 count $\mathrm{s}^{-1}$ pixel $^{-1}=$ $177 \mu \mathrm{Jy} \operatorname{arcsec}^{-2}=18.04 V_{\mathrm{mag}} \operatorname{arcsec}^{-2}$.

\section{SCATTERED-LIGHT IMAGE MODELING}

We ascertain estimates of the geometric, astrometric, and photometric characteristics of the debris rings themselves by comparing simple scattering models of the rings to best match their observed SB distributions (e.g., see Schneider et al. 2006). We assume an azimuthally uniform ring with a Gaussian radial distribution of the surface density of scattering particles and allow for offsets of the ring center from the star. We illuminate models with starlight according to an $r^{-2}$ power law and adopt a Henyey-Greenstein (HG) scattering phase function (Henyey \& Greenstein 1941). We treat the following as observationally informed free parameters, initially estimated visually and/or by ellipse fitting: the celestial position angle (P.A.), inclination $(i)$, and apparent semimajor axis (a) of a ring ellipse that is assumed to be intrinsically circular (but seen in projection). We also fit the inner and outer radii of the ring as characterized by the half-power points of their respective edge SB profiles, and the slopes of the inner and outer edges. We then additionally vary the two-dimensional displacement of the ring center from the stellocenter and the HG asymmetry parameter, $g$, through a grid of values with resolution 0.05 in $g$. In least-squares fitting the models to the data, regions both internal and external to the imaged debris rings, where PSF-subtraction and sky-plusinstrumental noise obviously dominates over detectable flux from the ring, were digitally masked. The free parameters of the model were then iterated to convergence, minimizing the variance in the fitting region. 


\section{INDIVIDUAL OBJECTS}

\subsection{HD 207129}

Introductory notes. HD 207129 is a G2V close-solar analog and is one of the two oldest stars (along with HD 202628; Section 7.2) for which a CDS has been imaged in scattered light. The presence of cold dust around HD 207129 was inferred by Walker \& Wolsterncroft (1988) from 12.5 to $60 \mu \mathrm{m}$ IRAS excesses, and confirmed by Jordin de Muizon et al. (1999) with 2.5-180 $\mu \mathrm{m} \mathrm{ISO} \mathrm{photometry} \mathrm{with} \mathrm{far-IR} \mathrm{excess} \mathrm{in}$ its spectral energy distribution (SED) at $>20 \mu \mathrm{m}$. A spatially extended disk was detected in thermal emission in Spitzer/ MIPS imaging at $70 \mu \mathrm{m}$ by Krist et al. (2010). Correcting for the broadening effects of the instrumental beam, they deduced an intrinsic size of an apparently elliptical disk at $70 \mu \mathrm{m}$ of 18 ." $8 \times 8$ " $1(300 \times 130 \mathrm{au})$. The disk, observed as part of the Herschel/DUNES program, has also been resolved with PACS imaging in thermal emission from 70 to $160 \mu \mathrm{m}$ (Marshall et al. 2011) as modeled by Lohne et al. (2012). Krist et al. (2010) additionally resolved the disk with HST/ACS visiblelight coronagraphy at $0.6 \mu \mathrm{m}$. The ACS image, derived from two-roll "self-subtraction" of the underlying stellar PSF, revealed a ring-like disk inclined $60^{\circ}$ from face-on with a major axis P.A. of $127^{\circ}$, in approximate geometrical agreement with the $70 \mu \mathrm{m}$ MIPS and Herschel/PACS images.

Observations and PSF subtraction (details). HST/STIS 6RPSFTSC observations of HD 207129, and its color-matched PSF-template star Tau ${ }^{1}$ Gru, were conducted according to the GO 13786 observing plan with no variances or anomalies on 2015 May 26 (visits 15-18) and 2015 August 8 (visits 11-14). With visit-level PSF subtraction, a low SB scattered-light excess from the disk was detectable (at low $\mathrm{S} / \mathrm{N}$ ) in each of the individual $1508 \mathrm{~s}$ exposure WedgeA-1.0 images, co-rotating with visit-to-visit reorientation of the celestial field. Despite the very close $|B-V|$ color-index matching of its PSF-template star, a stellocentric, (first-order) azimuthally symmetric, and radially modulated residual pattern (seen also independently in the WedgeA-0.6 images) at $r<6^{\prime \prime}$ was characteristically betrayed by a small chromatic error in PSF subtraction with stellocentric angle (undersubtraction at $r<1^{\prime \prime}$, oversubtraction at $1^{\prime \prime} \leqslant r \leqslant 3$ !'5, undersubtraction at $3{ }^{\prime \prime} 5 \leqslant r<6^{\prime \prime}$ ). We illustrate this (hard stretched for maximum visibility), uncorrected, after combining all images in a common celestial frame in Figure 1 panel (A). To largely mitigate this problem, we follow the empirical color-correction process detailed in Sch14 as applied therein by example for HD 92945 (with sourcemasking here appropriate for HD 207129); see Figure 1 panel (B). After correction, the WedgeA-1.0 deep images and WedgeA-0.6 shallow images, in combination, both well reveal the ring-like disk reported by Krist et al. (2010) and provide SB limits on any dust (or lack thereof) interior to the starlightscattering debris ring itself. For better visualization of the ringlike disk, in Figure 1 panel $(\mathrm{C})$ we convolve the color-corrected image with a $3 \times 3$ pixel boxcar smoothing kernel to reduce the pixel-to-pixel instrumental background noise.

Principal results. The STIS 6R-PSFTSC scattered-light imaging of the HD 207129 CDS (Figure 1) reveals an angularly large, ring-like disk of low SB at an intermediate inclination angle with a morphology as generally described by Krist et al. (2010). The broad ring-like disk appears to be devoid of starlight-scattering material interior to its inner edge to a stellocentric angle limit of $r \approx 5^{\prime \prime}$ where, at
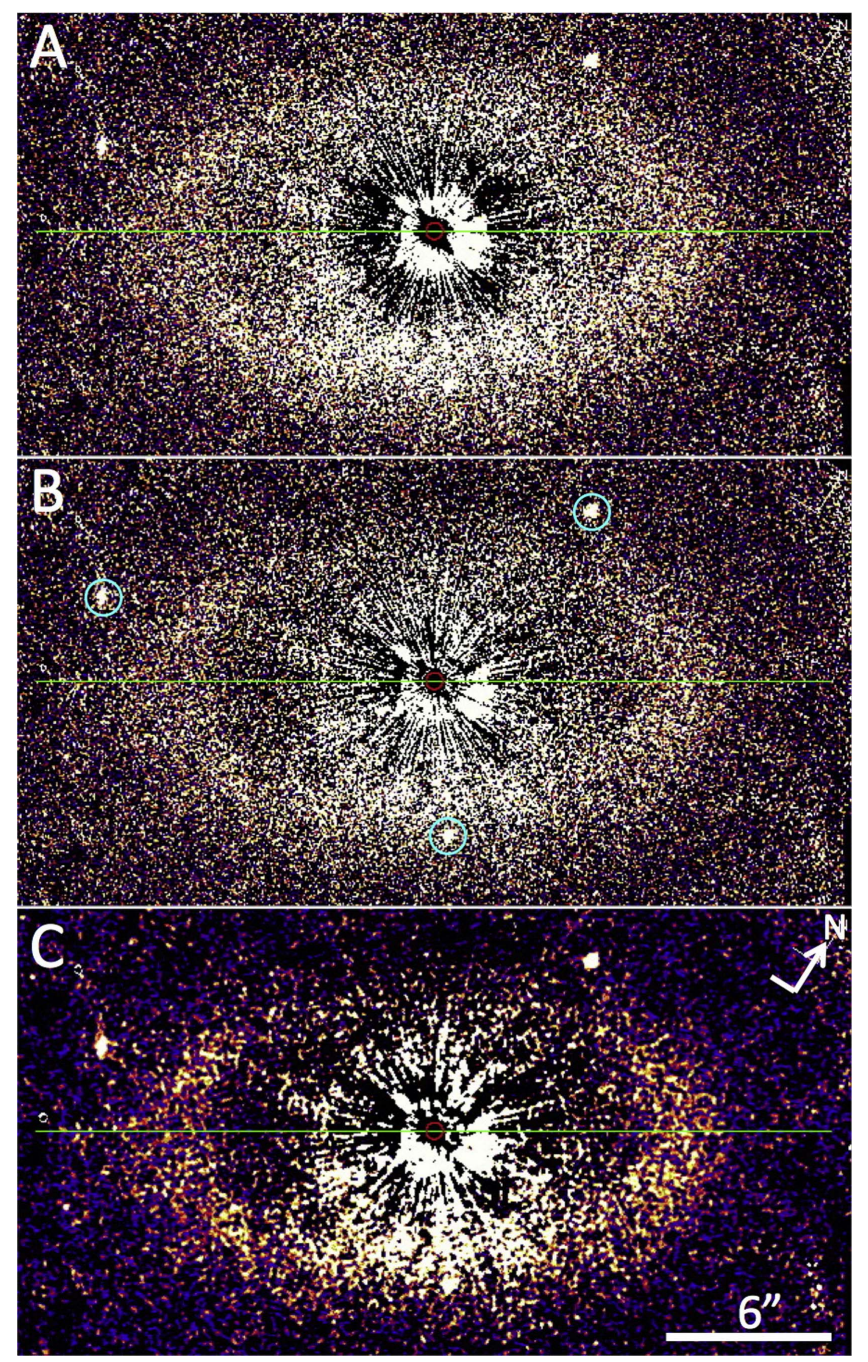

Figure 1. 6R-PSFTSC images of the HD 207129 CDS combining all WedgeA-0.6 and WedgeA-1.0 data, rotated $32^{\circ}$ clockwise from "north up" to place the morphological disk major axis on the image horizontal. (A) and (B) Respectively, before and after correcting for radially symmetric chromatic PSFsubtraction residuals. (C) Panel (B) convolved with a $3 \times 3$ pixel boxcar smoothing kernel to reduce pixel-to-pixel noise. Three compact/point sources (blue circles in panel (B)) identified by Krist et al. (2010) are confirmed as background objects through differential proper motions between the images from ACS epoch 2006 and STIS epoch 2015. All panels: field of view $(\mathrm{FOV})=25$ !" $4 \times 13$ ! 4 and linear display stretch from 0 to +0.015 counts $\mathrm{s}^{-1}$ pixel $^{-1}$.

smaller stellocentric angles, residual starlight dominates the centrosymmetric background. The SB ring model (Section 6) that best fits the observed data (by minimizing the sum of the squares of the observed-minus-model image residuals) is illustrated in Figure 2, with the model parameters given in Table 4. Although the model also allows for a stellocentric offset, none of significance was evidenced in initial fits to the HD 207129 image, and so it was fixed to zero, i.e., constraining the apparent ring center to be coincident with the location of the host star. We did not treat $g$ iteratively, but produced a grid of models incrementally increasing from $g=0.0$ (the isotropic case) in steps of $\Delta g=+0.05$, for which a best global fit was found for $g \sim 0.25$.

The model appears to give a very good fit overall, though may slightly oversubtract the disk flux asymmetrically with respect to the locations (radii) of the peak brightness at some 

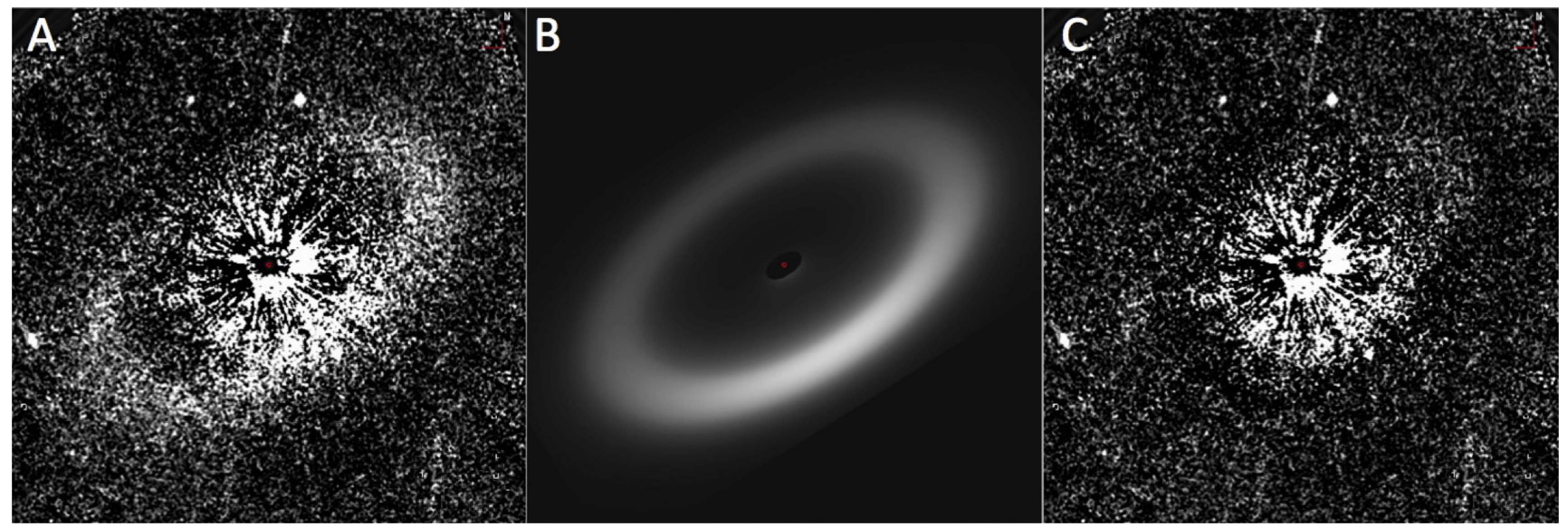

Figure 2. Best-fit debris ring model of HD 207917. (A) Observed image (north up). (B) Model with $g=+0.25$ and other parameters as in Table 4. (C) Observedminus-model image residuals. All images: linear stretch from -0.001 to +0.01 counts s $^{-1}$ pixel $^{-1}$, FOV $512 \times 512$ pixels $\left(26^{\prime \prime} \times 26^{\prime \prime}\right)$ with celestial north up and east left.

Table 4

Debris Ring Parameters for HD 207129

\begin{tabular}{|c|c|c|}
\hline $\begin{array}{l}\text { Inclination }(i) \\
\text { P.A. major axis }\end{array}$ & \multicolumn{2}{|c|}{ 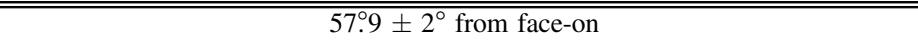 } \\
\hline Inner-edge radius of ring HWHM major axis & $7 ! 13 \pm 0 . \prime 04$ & $115.7 \pm 0.9 \mathrm{au}^{\mathrm{a}}$ \\
\hline Ring width (FWHM; $\Delta r)$ & \multirow{2}{*}{\multicolumn{2}{|c|}{$\begin{array}{c}72.3 \pm 1.5 \mathrm{au} \\
0.25 \pm 0.05\end{array}$}} \\
\hline$g$ (HG scattering asymmetry parameter) & & \\
\hline Total ring flux density ${ }^{\mathrm{a}}$ & $310 \pm 29$ counts s${ }^{-1}$ pixel $^{-1}$ & $141 \pm 13 \mu \mathrm{Jy}$ \\
\hline
\end{tabular}

Notes.

${ }^{\mathrm{a}}$ Includes uncertainty in stellar parallax from SIMBAD.

${ }^{\mathrm{b}} V_{\text {star }}=5.58 \rightarrow 17.1 \mathrm{Jy}$.

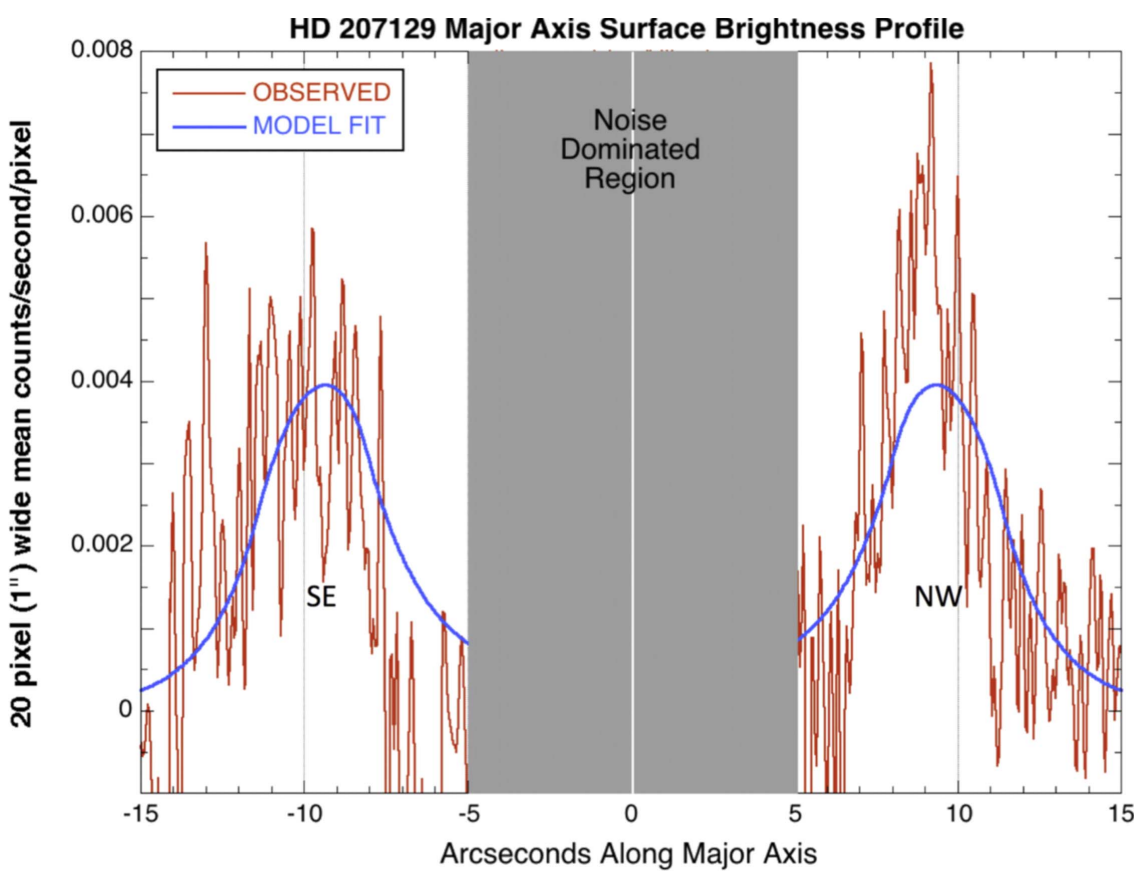

Figure 3. Radial SB profile of the HD 207129 CDS along the disk's major axis (red), and as modeled (blue) as in Figure 2 panel (B), with peak $\mathrm{SB} \sim 24 V_{\mathrm{mag}} \operatorname{arcsec}^{-2}$ at $\sim 9$ ". 5 . At $r \leqslant 5^{\prime \prime}$ residuals from stellar light, incompletely suppressed with imperfect PSF subtraction, dominate the stellocentric background, increasing in brightness from $\sim 23 V_{\mathrm{mag}} \operatorname{arcsec}^{-2}$ at $5^{\prime \prime}$ inward as illustrated in Figure 4. 
stellocentric azimuth angles. This is marginally evidenced in the somewhat darker (black) distorted "ring" of low-amplitude correlated negative-going residuals visible in Figure 2 panel (C). This may be indicative of the limitations of the current model, which does not account for possible anisotropies in the dust density or non-HG scattering properties.

1. Brightness and scattering fraction. In Figure 3 we compare the observed and model radial SB profiles along the disk's major axis where, at the ring ansae, the image contrast is less challenging and more accurately measured than closer to the minor axis. It is in this region that the deficit of light-scattering dust interior to the debris ring itself is visually apparent (e.g., in Figure 2 panel (A)). Using the model parameters in Table 4 closely replicates the main features of the observed profile on both sides of the star across the ring ansae, though some local deviations are seen-in particular here at $\sim 5^{\prime \prime}<r<7^{\prime \prime}$ where the model slightly overpredicts the observed brightness. This could be due to a deficiency in the model, or simply a bias in the signal as the stellocentric angle is approaching the noisedominated regime in the image at $r<5^{\prime \prime}$. The peak SB of the debris ring along its major axis, at its diametrically opposed ansae, appears marginally brighter on its northwestern side at $\sim 0.96 \mu \mathrm{Jy} \operatorname{arcsec}^{-2}\left(23.7 V_{\text {mag }} \operatorname{arcsec}^{-2}\right)$ and on its southeastern side $0.68 \mu \mathrm{Jy} \operatorname{arcsec}^{-2}\left(24.1 V_{\text {mag }} \operatorname{arcsec}^{-2}\right)$. The total $0.6 \mu \mathrm{m}$ flux density of the disk, as informed by the best-fit model, is $141 \pm 13 \mu \mathrm{Jy}$. This gives rise to a total disk optical scattering fraction, $F_{\text {disk }} / F_{\text {star }}$, of $\sim 8.2 \times 10^{-6}$ to its host star with $V_{\text {mag }}=+5.58$. This is in good agreement with the prior estimation by Krist et al. (2010) of $\sim 7.6 \times 10^{-6}$, independently derived with a different data set with iterative roll subtraction. Flux-loss and/or larger photometric uncertainties from "self-subtraction" for some disk geometries with this method are possible. However, the good agreement $(\approx \pm 5 \%)$ in the independently derived global scattering fraction for the HD 207129 CDS suggests that the original estimation by Krist et al. (2010) was photometrically well calibrated.

2. Anisotropic ("front/back") scattering asymmetry. From an analysis of the ACS imaging of the HD 207129 CDS from the 2006 epoch, Krist et al. (2010) suggested "at $0.6 \mu \mathrm{m}$, the ring shows no significant brightness asymmetry, implying little or no forward scattering by its constituent dust." They asserted $g<0.1$ with an interpretive consequence that the "nearly isotropic scattering in the ring conflicts with any assumption of spherical particles that scatter according to Mie theory" (separate from a low-albedo inconsistency for silicate grains). The STIS data, in contrast, inform directionally preferential anisotropic scattering that, with $g \approx 0.25$ from the best-fit modeling, is visually apparent as a "front/back" asymmetry (see Figure 2 panel (A)). In validation, though noisier, this same "front/back" asymmetry was seen in the STIS data when data from the first and second epochs were reduced separately into two independent three-roll images originating at different field orientation angles. The reason for this discrepancy in $g$ with the prior published ACS imaging is unclear. We speculate that it may be related to a lower $\mathrm{S} / \mathrm{N}$ (with shorter total integration time, narrower bandwidth, and lesser coronagraphic throughput) in the ACS data, or to the differing methods of reduction. The ACS image was derived from "roll subtraction," using the host star as its own PSF template. This obviates chromatic effects in PSF subtraction but, as noted previously, is subject to possible localized loss of disk flux from selfsubtraction, with the dependence on stellocentric distance

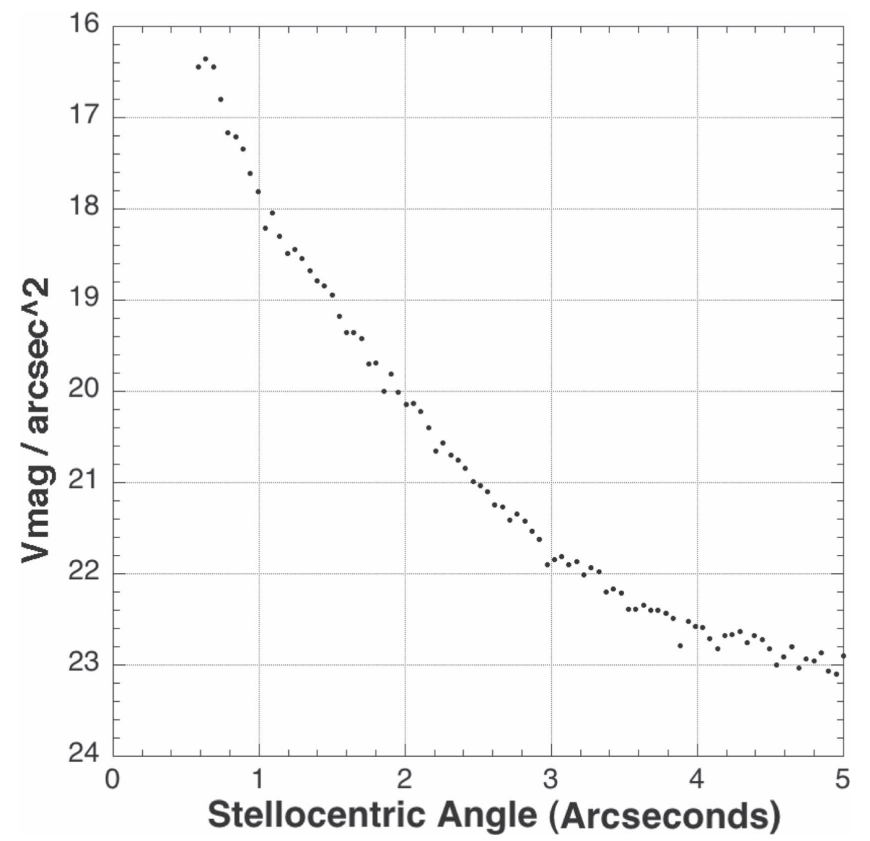

Figure 4. Limiting sensitivity determined by the median absolute deviation (MAD) for the detection of light-scattering debris dust as imaged in the inner stellocentric 5" of the HD 207129 CDS. Measured from the full set of colorcorrected PSF-subtracted images at each roll angle observed.

varying with azimuth angle, i.e., differently in the ring at the minor and major axes of the disk. The disparity may be more related to image structure (i.e., fidelity) than photometric efficacy, because both the STIS (six-roll) and ACS (two-roll) reduced data sets have relatively good agreement in the $0.6 \mu \mathrm{m}$ total disk flux density and average ansal SB.

3. Radial sensitivity to starlight-scattering material:

a. Interior (endo-ring) dust. We do not detect any lightscattering dust (either diffuse or in a secondary ring) comparable in brightness to the debris ring itself to an inner limiting stellocentric angle of $\sim 5^{\prime \prime}$. To quantitatively estimate the limits on imaging sensitivity to any (undetected) CS dust in the presence of incompletely suppressed starlight at, and interior to, this region, we plot in Figure 4 the $360^{\circ}$ median absolute deviation ${ }^{17}$ (MAD) in the color-corrected PSF subtraction residuals as a function of stellocentric angle after subtracting the debris ring model of Figure 2 panel (B). Very similar results are obtained with direct measurement of the stellocentric field, masking the regions along the disk's minor axis that are superimposed upon the centrosymmetric residual pattern.

b. Exterior (exo-ring) dust. We also do not detect any lightscattering material beyond the exo-ansal radial decay of SB as shown, e.g., along the disk's major axis in Figure 3. Beyond the ring seen in sky-plane projection, the backgrounds in the sectors toward and flanking its semiminor axes at $r \geqslant 9^{\prime \prime}-10^{\prime \prime}$ are wholly unaffected by the presence of the interior disk and the exterior is radially invariant. We directly assess the limits on imaging sensitivity in these regions (representative of

\footnotetext{
17 With a relatively small number of rolls (maximum six) contributing to each AQ image pixel, the MAD is a more robust (against outlier) estimator than the oft-used standard deviation; MAD $\approx 1.48 \sigma$ for large samples.
} 
fully circum-azimuthal measures) that are not dominated by PSF-subtraction residuals via multi-position aperture photometry as $\approx 25.8 \pm 0.3 V_{\text {mag }} \operatorname{arcsec}^{-2}$.

c. Intra-ring dust. $r \approx 9^{\prime \prime}-10^{\prime \prime}$ is also the distance to the ring peaks along the major axis, and so we approximately assess an intra-ring ansal average $\mathrm{S} / \mathrm{N} \approx 2.4 \operatorname{arcsec}^{-2}$.

\section{2. $H D 202628$}

Introductory notes. HD 202628 is one of 10 nearby $(<40 \mathrm{pc})$ solar analog (K0-G5) stars that were surveyed by Krist et al. (2012) for the presence of starlight-scattering CS dust potentially detectable with $H S T$ visible-light coronagraphy. This sample was IR-selected with Spitzer-derived excess thermal emission $L_{\mathrm{ir}} / L_{\mathrm{star}}>10^{-4}$, indicative of cold dust at stellocentric distances potentially within the imaging sensitivity capabilities of STIS for systems with favorable viewing geometries, dust properties, and ring-like morphologies with inner radii beyond the inner working angle limits of the coronagraph configuration employed. HD 202628 is a nearby $(24.4 \mathrm{pc})$ solar-type $(\mathrm{G} 2 \mathrm{~V})$ star with a thermal infrared excess $L_{\mathrm{ir}} / L_{\mathrm{star}}=1.4 \times 10^{-4}$ (Koerner et al. 2010) estimated from $70 \mu \mathrm{m}$ Spitzer/MIPS photometry, but lacking an excess at $24 \mu \mathrm{m}$, from which Krist et al. (2012) postulated an inner radius for the emissive dust of $80 \mathrm{au}\left(4{ }^{\prime \prime} 1\right)$. Of the 10 stars surveyed, HD 202628 was the only one for which a visiblelight debris system was imaged. The discovery imaging revealed a low-SB, intermediate-inclination, ring-like disk. Krist et al. estimated a semimajor axis length to the inner radius of the debris ring of $\sim 158 \mathrm{au}$, with characteristic width $\sim 58 \mathrm{au}$ averaged radially across both ansae, and noted a significant stellocentric offset.

Observations and PSF subtraction. On 2015 May 30 and September 17, HST/STIS coronagraphic images of the HD 202628 CDS were obtained to improve, and expand upon, the discovery-mode imaging of Krist et al. (2012). The discovery images were (a) obtained using STIS occulting Wedge-A at its 1 !. 8 wide location, (b) acquired in two HST orbits differing in a single orientation differential of $28^{\circ}$, (c) with total integration time $4512 \mathrm{~s}$, (d) without contemporaneously observed, nearby, color-matched PSF-template stars, and (e) reduced with tworoll "self-subtraction" of the underlying stellar PSF. The GO 13786 follow-up images were (a) obtained using STIS occulting wedge-A at a combination of its (narrower) $0 . " 6$ and 1". 0 wide locations, (b) acquired in six HST orbits with five field orientation differentials spanning $262^{\circ}$ (see Table 3), (c) with total integration time $12,670 \mathrm{~s}$, (d) with target-specific PSF reference stars, and (e) reduced with six-roll PSF-template subtraction.

All observations were executed as planned at both observational epochs. However, the preplanned PSF-template star for the first observational epoch (visit 07), BX Mic, was found to be "polluted" by third-light from an a priori unrecognized close-proximity companion (or ill-placed background star) rendering it unsuitable as a PSF-subtraction template. For the observations in the second epoch, the PSF-template star was changed to an alternative candidate, HR 8042, and found to be an excellent match. Fortuitously, PSF-subtraction using the second-epoch HD 8042 imaging showed little (if any) degradation due to possible differential breathing when applied to the first-epoch (visit 05, 06, 08) observations of HD 202628, and so was used as a PSF template for both sets of HD 202628

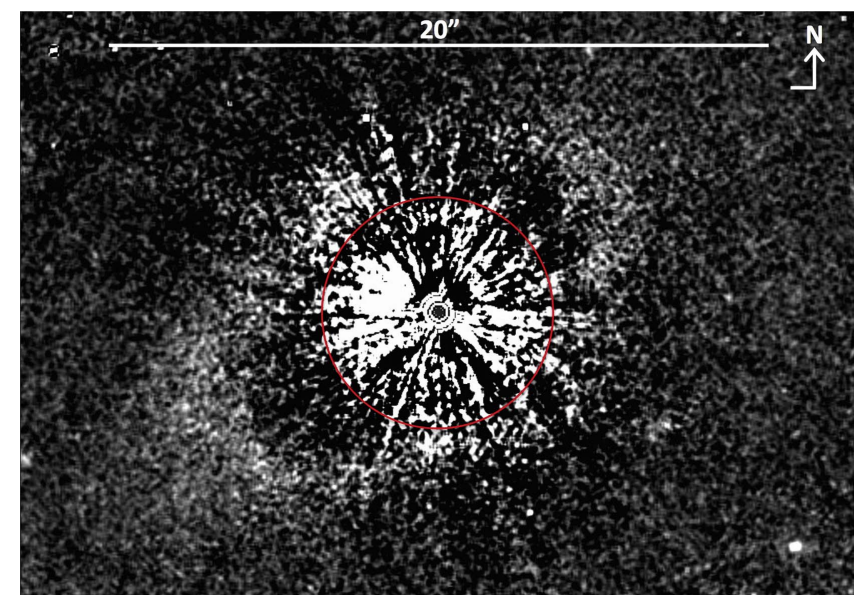

Figure 5. STIS 6R-PSFTSC image of the HD 202628 CDS. FOV $500 \times 350$ pixels $(25$ !" $4 \times 17$ !' 8$)$ centered on the host star. Linear display stretch -0.002 (hard black) to +0.009 (hard white) counts $\mathrm{s}^{-1}$ pixel $^{-1}$ with $3 \times 3$ boxcar smoothing to better illustrate the nature and amplitude of PSFsubtraction residuals interior to the debris ring, and structure of the background exterior to it. The red circle, with $r=3$ ! 5 , indicates the region within which stellocentric PSF-subtraction residuals closer to the star dominate over the brightness of the apparently elliptical debris ring observed at larger stellocentric angles.

visits. We applied the same method of image reduction, and two-wedge six-roll visit combinations of PSF-subtracted images as discussed for HD 207129 (Section 7.1), to produce the AQ data image (shown in Figure 5) discussed herein.

Principal results. The STIS 6R-PSFTSC AQ image of the HD 202628 CDS (Figure 5), from visual inspection, is generally in good morphological and photometric agreement with the discovery image of Krist et al. (2012) (see their Figure 2 top panel), though we note and discuss below some differences in details. The 6R-PSFTSC AQ image directly informs of the spatial distribution of the CS dust at all azimuth angles at $r>3$ ". 5-4". 0 , of greater apparent complexity than HD 207129. Principal geometric, photometric, and astrometric results discussed are summarized in Table 5.

1. Morphology. The HD 202628 CDS appears to comprise, in morphological description, two main components. First, dominating the system, a stellocentrically offset debris ring, and second, a low-SB diffuse "cloud" of exo-ring scattering material seen both exterior and somewhat interior to only the SE ansa.

a. The dust-scattered starlight from the ring appears to be mostly confined (within 50\%-peak SB inner and outer isophotes) in an elliptical annulus between $\sim 135$ and 218 au from the host star as seen in sky-plane projection. Interior to the ring, there is a notable deficit of scattering material in the two diametrically opposed sectors flanking the major axis $\left(\right.$ P.A. $=124^{\circ}$ ) from $17^{\circ}<$ P.A. $<158^{\circ}$ and $271^{\circ}<$ P.A. $<354^{\circ}$ to an inner working angle limit of $\sim 4^{\prime \prime}$ where residual starlight begins to dominate the background interior to the debris ring. The debris ring itself deviates from bilateral symmetry with its geometrical center offset from the location of its host star.

b. A notable low-SB excess of diffuse scattering material is seen externally to, and somewhat superimposed upon and internally to, the SE ansa (not apparent in the discovery imaging of Krist et al. 2012), but with none seen at or beyond the diametrically opposed NW ansa; see Figure 8 
Table 5

Debris Ring Parameters of HD 202628

\begin{tabular}{|c|c|c|c|c|}
\hline $\begin{array}{l}\text { Inclination }(i) \text { from face-on } \\
\text { P.A. of major axis of debris ring } \\
a \text { (elliptical annulus of peak SB) }\end{array}$ & \multicolumn{4}{|c|}{$\begin{array}{c}\left.54^{\circ} .2 \pm \sim 4^{\circ} \text { (axial ratio: } 1.708: 1\right) \\
124^{\circ} \pm \sim 3^{\circ}(\text { E of celestial } \mathrm{N})\end{array}$} \\
\hline $50 \%$ EDGES and PEAK RADII & \multicolumn{2}{|c|}{ From Star } & \multicolumn{2}{|c|}{ From Disk Center } \\
\hline NW inner edge (major axis) & 6". 409 & $156.4 \mathrm{au}$ & $5 ! 940$ & $144.9 \mathrm{au}$ \\
\hline Peak SB radius of NW major axis & $7 ! ! 628$ & $186.1 \mathrm{au}$ & 7!! 159 & $174.7 \mathrm{au}$ \\
\hline NW outer edge (major axis) & 8".948 & $218.3 \mathrm{au}$ & 8". 479 & $206.9 \mathrm{au}$ \\
\hline SE inner edge (major axis) & $5 " .522$ & $134.7 \mathrm{au}$ & 5".991 & $146.2 \mathrm{au}$ \\
\hline Peak $\mathrm{SB}$ radius of $\mathrm{SE}$ major axis & $6 ! 740$ & $164.5 \mathrm{au}$ & $7 ! ! 209$ & $179.5 \mathrm{au}$ \\
\hline SE outer edge (major axis) & $8 ! \cdot 060$ & $196.7 \mathrm{au}$ & $8 ! .529$ & $209.9 \mathrm{au}$ \\
\hline SB SLOPES (Power-law Index) & \multicolumn{2}{|c|}{ SE (Disk-centered) } & \multicolumn{2}{|c|}{ NW (Disk-centered) } \\
\hline 142 au $<R<158$ au (inner) & \multicolumn{2}{|c|}{+12.6} & \multicolumn{2}{|c|}{+7.7} \\
\hline 175 au $<R<200$ au (outer) & \multicolumn{2}{|c|}{-4.5} & \multicolumn{2}{|c|}{-12.9} \\
\hline 200 au $<R<250$ au (outer) & \multicolumn{2}{|c|}{-3.9} & \multicolumn{2}{|c|}{-2.8} \\
\hline 250 au $<R<300$ au (outer) & \multicolumn{2}{|c|}{-6.9} & \multicolumn{2}{|c|}{$\cdots$} \\
\hline NW Ring width (FWHM; $\Delta r$ ) & \multicolumn{4}{|c|}{$2: .539 ; 60.0 \mathrm{au}$} \\
\hline$g$ (HG scattering asymmetry) & \multicolumn{4}{|c|}{$0.1 \pm 0.1$} \\
\hline Stellocentric offset from ring center in sky-plane projection ${ }^{\mathrm{a}}$ & \multirow{2}{*}{\multicolumn{4}{|c|}{$\begin{array}{c}+0 . " 469(\Delta X),-0 . \prime 277(\Delta Y)=0 . " 535 \text { toward ring-azimuth } 239.4 \\
0 . ! 68316.7 \text { au }\end{array}$}} \\
\hline Stellocentric offset from ring center in face-on deprojection & & & & \\
\hline SB @ NW ansa & \multicolumn{2}{|c|}{$0.40 \mu \mathrm{Jy} \operatorname{arcsec}^{-2}$} & \multicolumn{2}{|c|}{$24.6 V_{\mathrm{mag}} \operatorname{arcsec}^{-2}$} \\
\hline SB @ SE ansa & \multicolumn{2}{|c|}{$0.51 \mu \mathrm{Jy} \operatorname{arcsec}^{-2}$} & \multicolumn{2}{|c|}{$24.4 V_{\mathrm{mag}} \operatorname{arcsec}^{-2}$} \\
\hline Total CDS flux density $\left(F_{\text {disk }}\right)$ & \multirow{2}{*}{\multicolumn{4}{|c|}{$\begin{array}{l}44.6 \pm \sim 9 \mu \mathrm{Jy} \\
7.65 \times 10^{-6}\end{array}$}} \\
\hline Disk $(0.6 \mu \mathrm{m})$ scattering fraction $\left(F_{\text {disk }} / F_{\text {star }}\right)$ & & & & \\
\hline
\end{tabular}

Note.

a Measured along the disk's major $(X)$ and minor $(Y)$ axes. panel (B). This "cloud" of material has a comparatively very shallow outer slope, leaving the outer "edge" of the ring beyond the SE ansa not as sharp or well defined as for the NW ansa. It may also apparently "intrude" (in morphological superposition) a short distance interior to the brightest part of the ring itself. The eastern sector of the ring between the $\mathrm{NE}$ minor axis (at P.A. $=34^{\circ}$ ) counterclockwise to near the SE ansa is marginally brighter (and/or wider at comparable brightness) than the diametrically opposed sector.

c. Krist et al. (2012) found the SB of the ring's SE ansa itself about half as bright as the NW ansa. This is an anisotropy that is not reproduced in the follow-up imaging presented here (e.g., see the radial profile of the major axis in Figure 7). We speculate that the previously reported ansal SB asymmetry and dearth of detectable exo-ring material beyond the SE ansa in the discovery imaging may have been a consequence of two orientation roll-subtraction partially cancelling the diffuse dust signal at these conjoined locations. This conjecture may eventually be tested with additional multi-roll imaging from HST GO program 13455 (J. Krist 2015, private communication).

d. As is also discernable in the prior discovery imaging, the apparent geometrical center of the debris ring in the follow-up imaging is offset to the east (toward P.A. $\sim 60^{\circ}$ ) from the location of its stellar host. However, in the follow-up imaging no (expected) corresponding pericentric "glow" is seen. This suggests the possibility of an azimuthally nonuniform dust density distribution (with or without anisotropic scattering) that, elsewhere, may be also locally implied by the presence of the SE primarily exoansal diffuse cloud of material.
2. Geometry. Beginning with a first visual estimation of the debris-ring geometry from the AQ image, we then parametrically fitted the ring-like component of the CDS to the same model described in Section 6. Our best fits to the simple ring models, illustrated in Figure 6, inform a less steep intermediate inclination of $\sim 54^{\circ}$ from face-on, as opposed to $\sim 64^{\circ}$ estimated previously by Krist et al. (2012) from apparent major:minor axial length ratios. These authors noted that in the discovery imaging most of the disk in the direction of its minor axis was obscured (remediated in the follow-up six-roll imaging reported here). We conjecture that with this lack of additional information there may have been a bias in the original estimation of the inclination. We also note a small $\left(\sim 6^{\circ}\right)$ difference with the prior estimation of the celestial position angle of the disk's major axis, derived as a free parameter in visual ellipse fitting with an a priori adopted inclination that had been fitted only to the inner edge of the elliptical annulus in the discovery imaging. While no formal errors were given by Krist et al. (2012) for these, and other estimated parameters, they note that reliable least-squares fitting was precluded due to low S/N. Given the above we (somewhat subjectively) do not find these differences discrepant with significance.

3. Stellocentric offset. The originating set of GO 13786 images at each of the six field orientation angles, before PSF subtraction, record the HST diffraction spikes centered on the occulted star. Following Sch14, using the "X marks the spot" diffraction-spike fitting method, we are readily able to determine the location of the occulted star in the 6R-PSFTSC AQ image with an uncertainty of $\sim \pm 4$ mas rms. We directly compare this to the geometrical center of the best-fit ring models to ascertain the presence and amount of a stellocentric offset of the debris ring that may inform a forced eccentricity (e.g., by an unseen planet). Krist et al. (2012) identified such an 

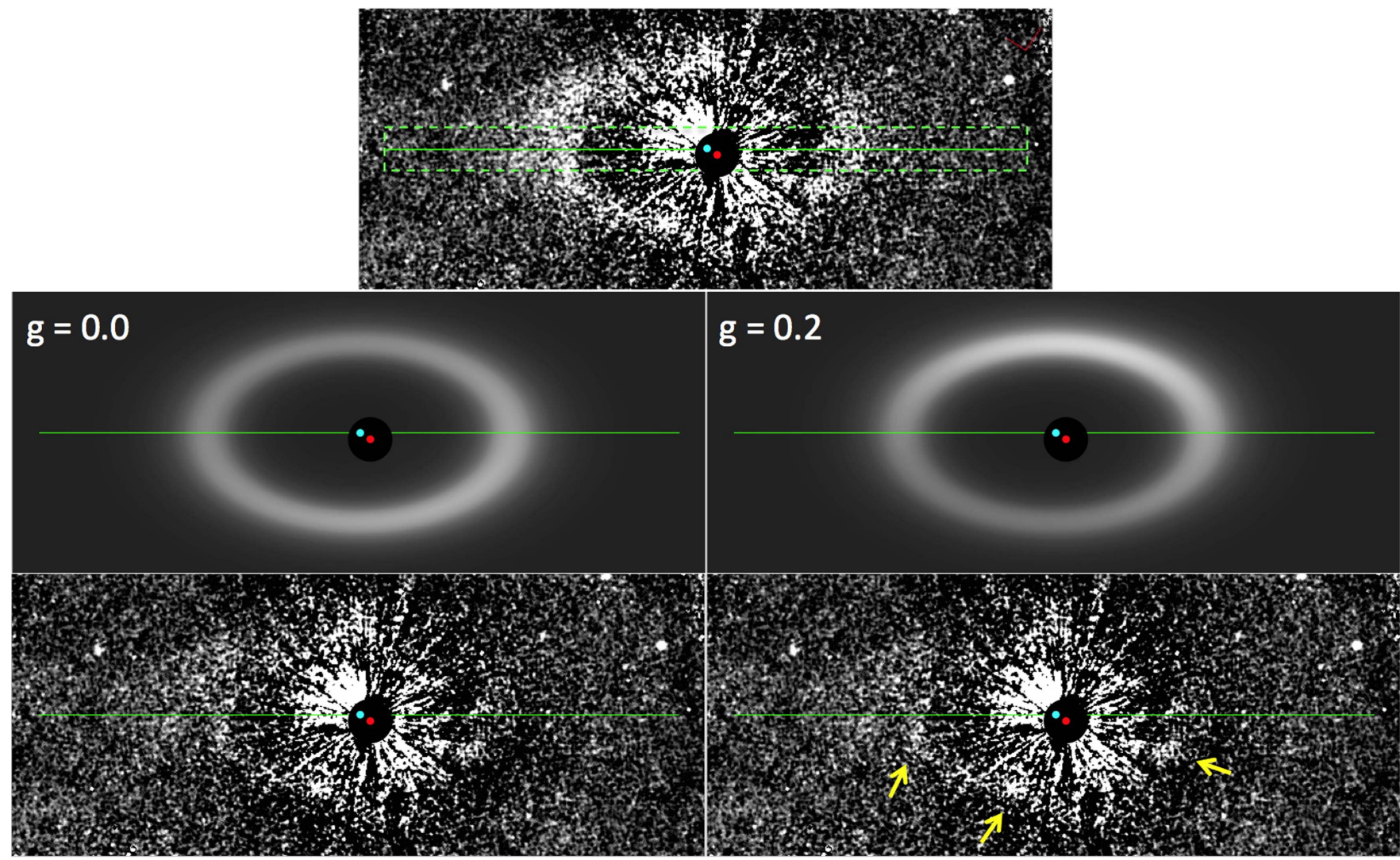

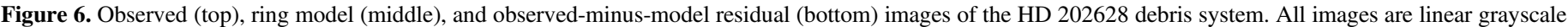

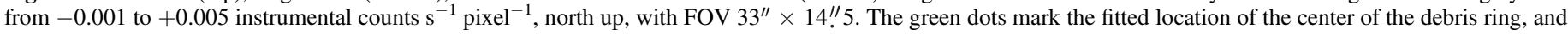

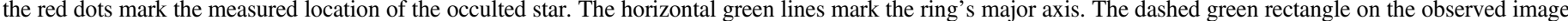

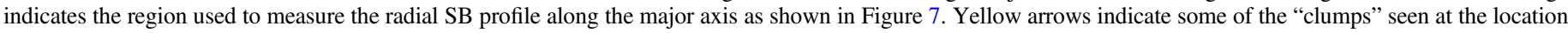
of the debris ring after subtracting the ring model.

offset in the discovery imaging estimated from ellipse fits to the inner edges of the debris ring (only). We confirm such an offset from the follow-up imaging wherein we find from our best-fit ring model the apparent geometrical center of the debris ring offset from the star by $(\Delta X, \Delta Y)=[-0.47,+0$." 28] (measured in a Cartesian coordinate system defined by the major and minor axes of the debris ring seen in sky-plane projection), i.e., an apparent offset of 0 !"545 toward P.A. $=239^{\circ} .4$. With sky-plane deprojection, assuming an inclination of $54^{\circ} .2$ from face on, this is a deprojected offset of 0. " 67 (16.4 au). This offset, determined in fitting the entire debris ring ellipse, while closely agreeing in celestial P.A., is smaller than estimated by Krist et al. (2012) using the inner edge only in the discovery imaging.

4. (Nearly) isotropic scattering. With an assumption of an azimuthally uniform dust density distribution, a pericentric brightening of the debris ring of $\approx+13 \%$ is expected toward P.A. $\approx 240^{\circ}$ (the direction from the center of the debris ring toward the host star), and is encoded in the isotropic $(g=0.0)$ scattering model illustrated in Figure 6 (middle panels). This, however, is not seen in the observed image (top panel), and indeed it is the diametrically opposed sector toward P.A. $\approx 60^{\circ}$ that is marginally brighter. Such a brightening, independent of the $r^{-2}$ scaling expected with offset stellocentric distances, suggests the possibility of an enhancement in dust density to the NE (though we cannot rule out some contribution to the possibility of some directionally preferential scattering). This is also consistent with contiguously adjacent brightening by the low SB "cloud" of material seen beyond the eastern ansa and may (or may not) be a physically contiguous feature. The ring itself is relatively well fit, over the explorable range of scattering phase angles, assuming nearly isotropic HenyeyGreenstein scattering, $g=0.1 \pm 0.1$, with the forward scattering direction along the ring's minor axis to the NE with residuals shown for both flanking cases in Figure 6 (bottom panels). After subtracting the $g=0.0$ model, the residuals along the ring are enhanced relative to $g=0.2$ to the north of the star (toward scattering phase angle zero) and diminished to the south, and vice versa. (This is readily apparent when "blinking" between these two images.) Those residuals, however, are (globally) not fully eliminated with intermediate asymmetry parameter values also modeled. In any case, the residuals to the SE of the star, near and beyond the SE ring ansa are (not surprisingly) dominated by excess light from the previously discussed exo-ring "cloud" that is not included in the simple ring model.

5. "Clumpiness" along the ring? A careful examination of the spatial distribution of the residuals in the location of the ring, after subtracting the best-fit model, reveals some "clumpy" substructures, in particular along its southern side (see Figure 6 bottom panel) on multi-pixel spatial scales that one would not expect from an unperturbed (smooth) dust density distribution. These clumps may simply be imaging artifacts at the faint limit of image fidelity - though comparable posited artifacts are not seen elsewhere in the field - or possibly confusion from ill-placed background sources. In principle, 


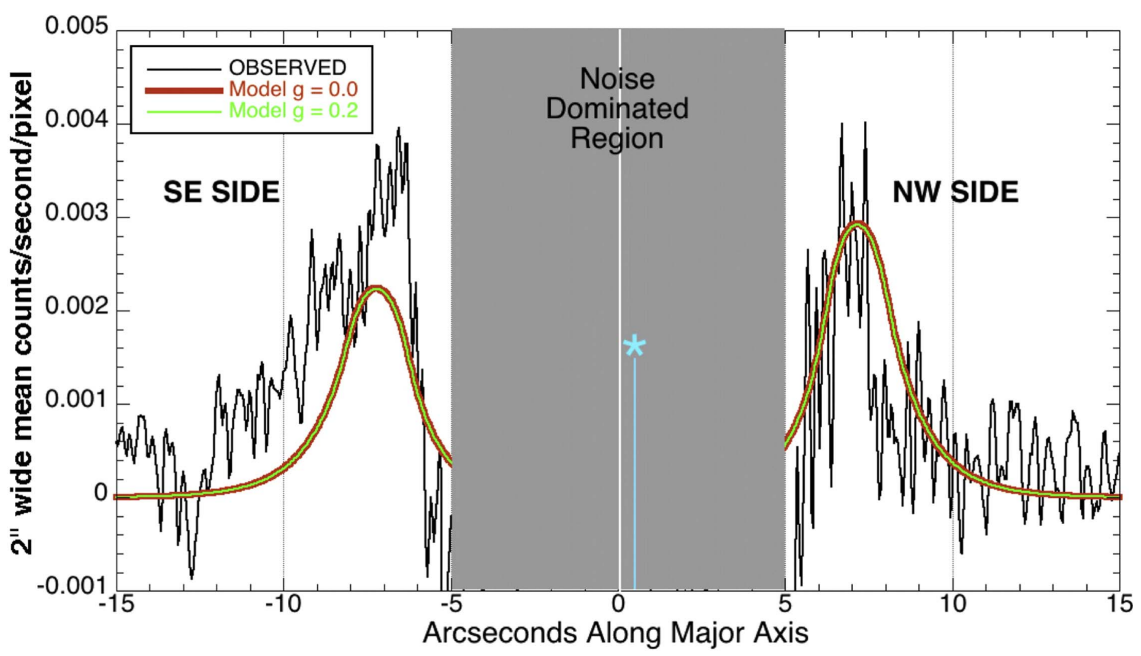

Figure 7. Radial SB profile of the debris ring of HD 202628 with respect to the geometrical center of the ring ellipse along its apparent (sky-plane projected) major axis-with models constrained to fit the peak intensity on the NW side of the star (see text).

with four years of stellar proper motion of $\sim 1$ !" 0 , the discovery image of Krist et al. (2012) could be used to arbitrate the latter. However, the same substructure pattern is not apparent in a discovery-epoch, roll-subtraction, image (see their Figure 2 top panel). This is likely due (if "real") to a combination of poor roll-angle coverage (wedge obscuration) in the sector(s) flanking the disk's minor axis, and differences in imaging efficacy with roll-subtraction versus multi-roll PSF-template subtraction. At this time a possible intrinsic origin for the intraring substructures seen in the follow-up images cannot be confirmed, but is testable with comparable (or improved) thirdepoch imaging in the future.

6. Radial SB profile of the major axis. The major axis radial SB profile of the CDS, presented in Figure 7 (black line), was measured along the disk's major axis in both directions extending $\pm 15^{\prime \prime}$ ( $\sim 300$ pixels) from the center of the debris ring. The rectangular measurement region with its long dimension parallel to the major axis of the debris ring is depicted in Figure 6 (top panel). Along that strip we measured the average flux density in bins one pixel wide and \pm 20 pixels in extent orthogonal to the disk's major axis, at stellocentric angles $>5^{\prime \prime}$ (beyond the central noise-dominated region). In Figure 7 we also overplotted the identically measured majoraxis radial SB profiles of the best-fit ring models previously discussed with characterizing parameters given in Table 5 . Along the major axis (and therein through the diametrically opposed ansae) the difference in the $g=0.0$ and $g=0.2$ models is indistinguishable at the resolution of Figure 7. Here, we constrained the models to reproduce (scale) the SB of the ring at the NW ansa. The $\sim 26 \%$ difference in the predicted diametrically opposing ansal flux densities arises from the stellocentric offset of the debris ring. The NW side of the ring along the major axis is well fit to the model (with a noted deviation, however, at 7!"5<r<8!!7). The model, in the presence of the exo-cloud clump on the SE side of the CDS, underpredicts the $\mathrm{SB}$, though the inner edge location (defined by the $50 \%$ intensity point) and outer "edge" slope (though offset in brightness) are well matched to the observations.

7. Ring width and edge slopes. Following Schneider et al. (1999) (in the case of HR 4796A), we characterize and measure the inner and outer radii of the debris ring along its major axis from the locations where the radial SB has declined to $50 \%$ of its peak. We additionally estimate the SB gradient across the inner edge of the ring, i.e., its slope, fit with a radial power-law dependence along the major axis of the debris ring. For these (steep) inner edge slopes, we fit radial regions from 142 to 158 au ( $\sim 5$ !" 8 to 6". 5$)$ on both sides of the star centered on the disk. Radially beyond the radii of peak brightness, we fit separately in three contiguous regions between 175 and 300 au (see Table 5). These metrics, in combination with the ring diameter, may be used to set constraints on the locations and masses of unseen planets that may be responsible for the ring architecture, e.g., see Rodigas et al. (2014b). The presence of the low-SB cloud of material producing additional scattered light at and beyond the SE ring ansa biases the measurements of the adjoining outer slope and thus width, and should be viewed with caution. Physical modeling, beyond the scope of this current paper, is necessary to disentangle the two.

8. Exo-ring extent. Figure 7 also shows that the radial SB profiles of the major axis across both ring ansae are comparatively asymmetric, in particular beyond the radius of peak brightness. The observations inform (different from Krist et al. 2012) similar ansal peak SBs on opposite sides of the disk. Except for a local deficit between 7!"4 and 8."5, on the NW side the model reproduces the outer-edge slope and fall-off in brightness to the level of the sky noise at $r<11^{\prime \prime}(\sim 270 \mathrm{au})$. On the SE side of the disk, in the presence of a local enhancement in exo-ring brightness visible in Figure 5, the model underpredicts the brightness beyond the ring ansa to $\sim 12$ ". 5 ( $\sim 305 \mathrm{au}$ ) on (and flanking) the major axis. This may be evidence of exo-ring scattering material seen preferentially only beyond the NE ansa. Speculatively, such material may arise from the launching of small grains after an episodic collision, or from density perturbations ("confinement" by resonances) by unseen planet(s). Higher (still) $\mathrm{S} / \mathrm{N}$ observations to better constrain the PSF would be beneficial to disambiguate the possibilities.

9. Total disk flux density and scattering fraction. Following chromatic correction in PSF subtraction, a faint, but not insignificant, azimuthally and centrosymmetric negative residual, diminishing with stellocentric distance, remains present beyond the $\sim 3 ! \prime 5$ radius where highly structured PSFsubtraction residuals otherwise dominate the background (see Figure 8, panel (A), dark area beyond the digital mask). The 


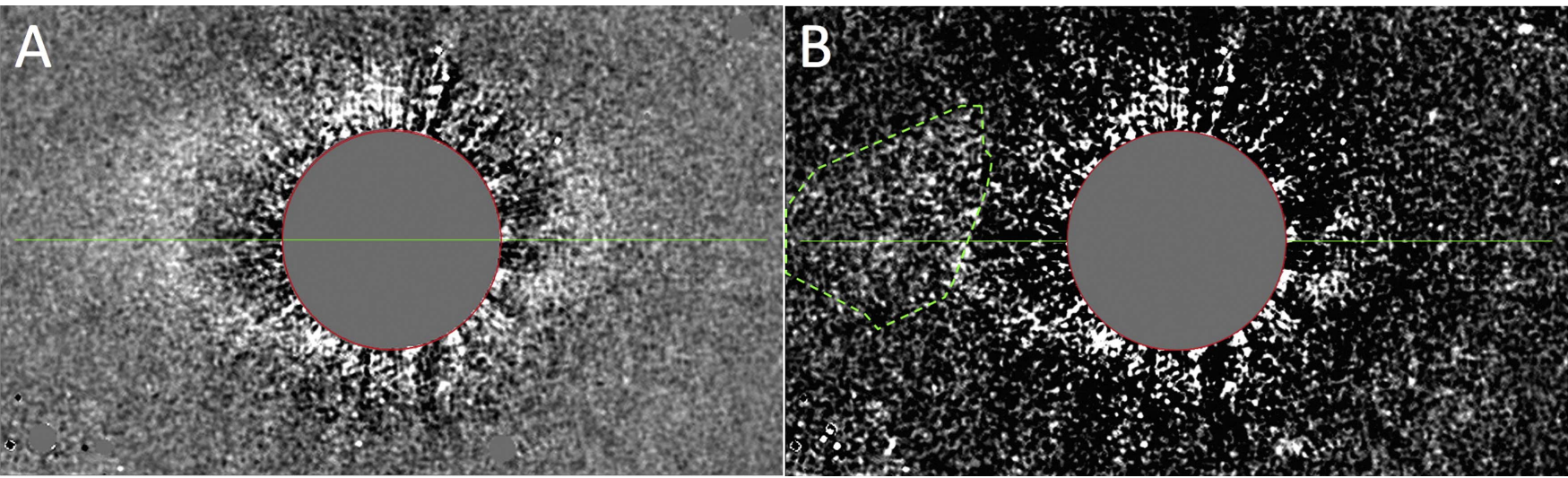

Figure 8. (A) Enclosing region used to estimate the flux density of the debris ring in the presence of a residual chromatic bias (oversubtraction) in the stellocentric background with the $r \leqslant 70$ pixel region dominated by PSF-subtraction residuals (shown in Figure 5) digitally masked. $500 \times 300$ pixels ( 25 !' $3 \times 15$ !'2; north up). The range of the linear grayscale display is \pm 0.009 counts s$^{-1}$ pixel $^{-1}$ to best show the background bias. (B) After subtracting the best-fit model (stretch: -0.001 to +0.005 counts s${ }^{-1}$ pixel $^{-1}$ ) the region used to assess the flux density in the exo-ansal SE cloud is marked by the dashed green line.
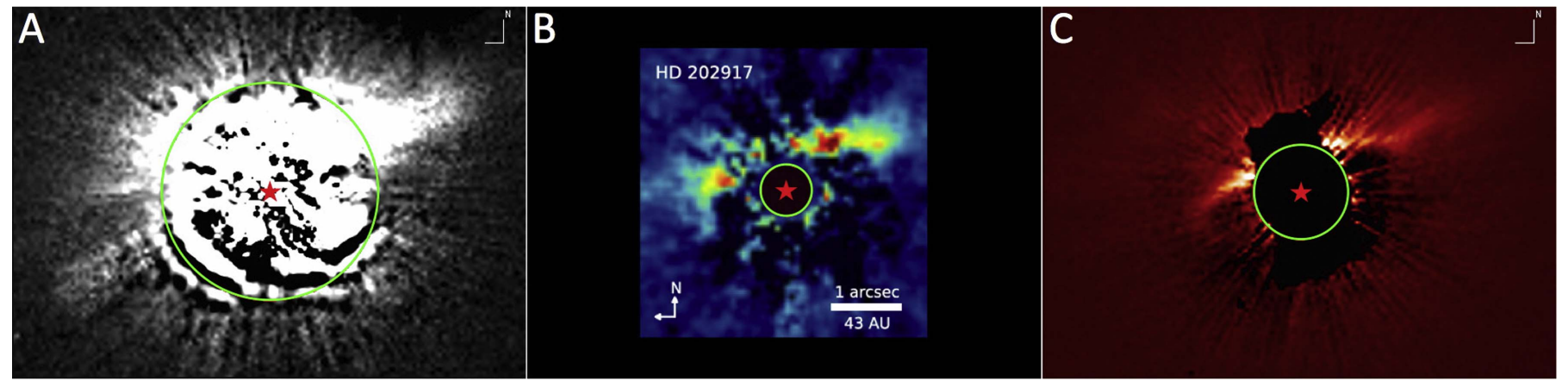

Figure 9. HD 202917 CDS. (A) ACS F606W ("wide V" band) from reprocessed archival data from HST program 10695. Scattered-light excess above the level of PSF-subtraction residuals is seen to the north of the star at $r>1$ !"5. (B) NICMOS F110W (1.1 $\mu \mathrm{m}$ ) from Soummer et al. (2014). (C) STIS unfiltered (optical broadband) with 6R-PSFTSC. Green circles indicate effective inner working angles: $r=1$ !. 5,0 " 36,0 ." 66 , respectively.

innermost $r<3$ ".5 region (shown in Figure 5) is digitally masked in making direct photometric measurements of the CDS. However, the presence of this remaining radial bias in the stellocentric background exterior, though of only minor consequence at the locations of the ring ansae at $r \sim 7$ !'2, correspondingly biases aperture photometry by its inclusion in the directions of and flanking the ring's semiminor axes of length $r \sim 4$ !"2. We therefore use the best-fit parametric model of the ring to estimate its total brightness, exclusive of the SE exo-ansal extension that is not included in the ring model. We refit with the same geometrical parameters of the ring, but to separately match the brightness of the ring in only the diametrically opposed $90^{\circ}$ sectors flanking the major axis, where the radial background gradient is a much smaller bias. In this manner we roughly estimate that the full-ring fit underestimates the total ring flux by $\sim 30 \%$ in total flux density. With this systematic correction we then estimate the total instrumental brightness of the debris ring as 88 counts $\mathrm{s}^{-1} \pm \sim 20 \%$ or $(4.0 \pm 0.8) \times 10^{-5} \mathrm{Jy}$. This does not include the additional light scattered by the SE exo-ring "cloud." To estimate that contribution to the total brightness we subtract the best-fit ring model from the observed image and directly measure the (somewhat subjectively bounded as shown in Figure 8) region contributing an additional $\sim(4.6 \pm 0.9) \times 10^{-6} \mu \mathrm{Jy}$, i.e., $F_{\text {disk }}=45 \pm \sim 9 \mu \mathrm{Jy}$. We adopt a catalog $V_{\text {mag }}$ for $\mathrm{HD}$ 202628 of $6.75 \mathrm{Jy}$ ( $5.83 \mathrm{Jy}$ in the STIS 50CCD band), from which the optical scattering fraction of the disk is $F_{\text {disk }} /$ $F_{\text {star }} \approx 7.7 \times 10^{-6}$, for spectrally flat gains.
10. $\mathrm{SB}$ of the debris ring. We characterize the representative SB of the CDS, independent of asymmetries expected from a stellocentric offset of the debris ring and anisotropic scattering, by measuring and averaging the SB at both ansae. We do so in circular regions, $1 \operatorname{arcsec}^{2}$ in area, centered on the radial SB peaks at the ring ansae. At this stellocentric distance along the disk's major axis $(\sim 7 ! 2)$ the residual chromatic error in the background that is corrected for in the estimation of the total disk flux is small to negligible. We find (averaging the two ansal brightnesses, which differ by $\pm 12 \%$ with the NW side brighter) a mean SB of $\sim 0.46 \mu \mathrm{Jy} \operatorname{arcsec}^{-2}$ (or $\sim 24.5 V_{\text {mag }} \operatorname{arcsec}^{-2}$ ).

\section{3. $H D 202917$}

Introductory notes. HD 202917 (HIP 105388; G7V, 43.0 pc) is a member of the Tucana-Horologium stellar association with an estimated age of 10-40 Myr (Moór et al. 2006). The presence of a thermally emissive CDS associated with HD 202917 was first suggested by Silverstone (2000) from 60-100 $\mu \mathrm{m}$ ISOphot data, and later confirmed from its $\sim 3 \times 10^{-4}$ infrared excess determined from Spitzer/MIPS observations (Beichman et al. 2005). An asymmetric scatteredlight excess to the north (only) of the star was suggested by Krist (2007) in "wide" V-band observations obtained in 2005 with ACS coronagraphy (HST program 10695) sensitive to dust-scattered starlight at stellocentric angular distance $\gtrsim 1$ !"5; see Figure 9 panel (A) where we have independently reduced those raw data obtained from the MAST archive with template 

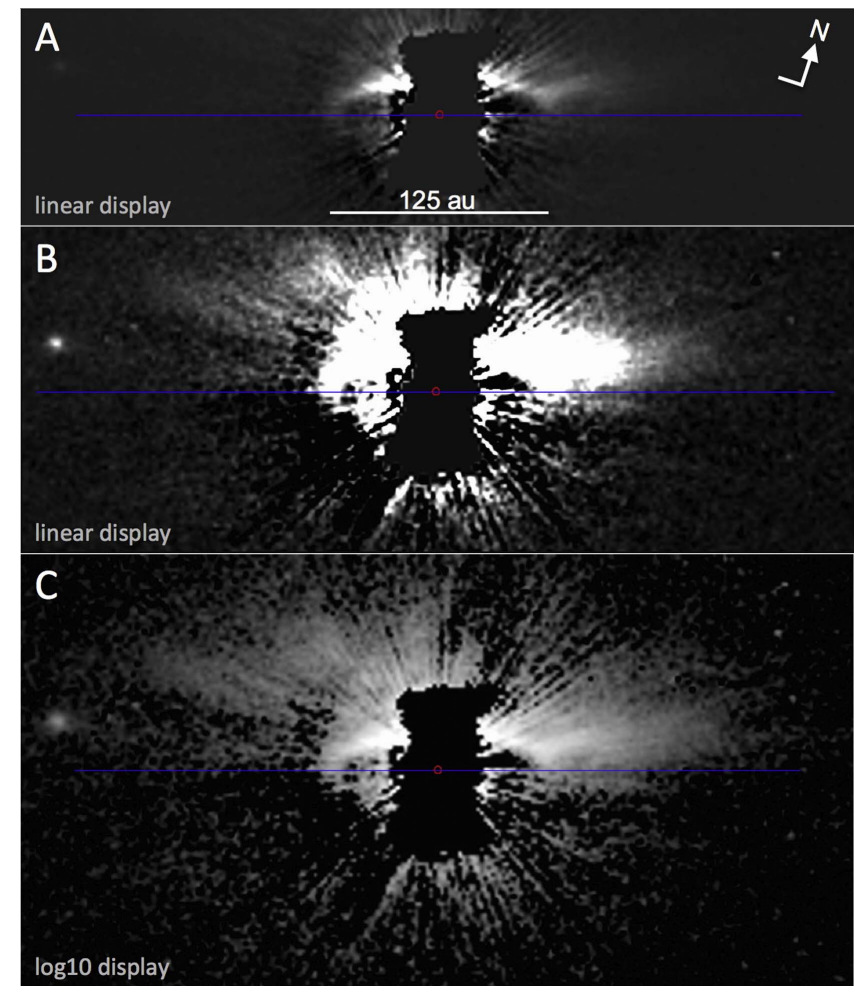

Figure 10. Highly asymmetric morphology of the debris ring of HD 202917 and its exo-ring "skirt," both preferentially scattering (and seen) on only one side of the ring's major axis, is illustrated over a large range of $\sim 300 \times$ in SB in three display images (same data). Panel (A) from -0.01 to +0.1 counts $\mathrm{s}^{-1}$ pixel $^{-1}$ (FOV: 11!' $67 \times 3$ !'05), panel (B) from -0.2 to +1.0 counts s $^{-1}$ pixel $^{-1}$ (FOV: $11^{\prime \prime} 67 \times 4$ ". 57), panel (C) from [-2.5] to [0] dex counts s ${ }^{-1}$ pixel $^{-1}$ (FOV: 11". $67 \times 6$ ". 09 ).

PSF subtraction. Earlier (1999 in HST GO program 7226), NICMOS $1.6 \mu \mathrm{m}$ coronagraphic images of HD 202917, designed for point-source (giant planet companion) detection by two-roll "self" subtraction, were obtained without PSFtemplate observations. Later (2005 in HST GO program 10849; one of 21 targets), images of HD 202917 at $1.1 \mu \mathrm{m}$ were acquired for disk detection, but without a contemporaneous or specifically programmed PSF-template star. In neither case was a scattered-light excess from a CDS detected. More recently, Soummer et al. (2014) re-reduced those prior data with principal component analysis (PCA) using a now-available extensive suite of PSF reference data from the MAST-hosted ${ }^{18}$ (NICMOS coronagraphic) Legacy Archive PSF Library (Schneider et al. 2011). The PCA reduction of Soummer et al. at $1.1 \mu \mathrm{m}$ (which provides the best NICMOS spatial resolution) is reproduced in Figure 9 panel (B), wherein the northern "half" of the disk (well correlated in position with the ACS image) was detected. From this image morphology, Soummer et al. (2014) suggested that the "disk exhibits an asymmetric arc suggestive of a partial ring inclined $\sim 70^{\circ}$ to the line of sight with a major axis P.A. $\approx 300^{\circ}\left[=120^{\circ}\right]^{\prime \prime}$, also noting that "the northwest side is significantly brighter and more extended than the southeast side." Our new STIS PSFTSC imaging (Figure 9 panel (C) and Figure 10) confirms these findings while providing both a higher-fidelity view of

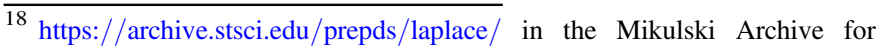
Space Telescopes (MAST). the ring-like disk and deconflating now well-seen exo-ring material from light scattered by the debris ring itself.

Observations and PSF subtraction (details). WedgeA-1.0: (a) All coronagraphic observations of HD 202917 from both the 2015 August 1 epoch (visits 55-58) and October 13 epoch (visits 51-54) were executed nominally as planned with no absolute constraints imposed on orientation. The observations of the WedgeA-1.0 PSF template of HD 8893 obtained contemporaneously (as visit 57) with the August 1 CDS images, however, were too deeply exposed. As a result, those images were saturated to larger-than-planned stellocentric angles in the directions orthogonal to the mid-line of WedgeA to $r \sim 1$ "! 1 (22 pixels), and so do not, with application, provide information on the CDS at distances closer than this. However, the serendipitous absolute orientations for the relative roll constraints in these visits (but not the October 13 visits) had the Wedge-A itself obscuring the disk itself (so would not have been visible in these data in any case). (b) Exposure times for the HD 8893 PSF template visit (\#53) of the second epoch were correctly adjusted prior to execution to circumvent saturation at $r>0$ ".5 (10 pixels). With that, in the PSF-subtracted images of the second epoch (only), the ring-like disk is seen protruding on opposite sides of the Wedge-A at all three image orientations with a revealed major axis ring radius of $\sim 1$ !" 5 (29.5 pixels).

WEDGEA-0.6: (c) The debris ring itself is also seen, but with inferior fidelity, in the less deeply exposed WedgeA-0.6 PSF-subtracted imaging for the more favorable wedge orientations of (second epoch) visits 51-54. With their PSF subtraction residuals, they contribute only marginally to improving the Inner Working Angle (IWA) over WedgeA1.0 imaging from these visits. (d) Separately, the WedgeA-0.6 PSF-template star in visit 57 of the first epoch (unfavorable wedge orientations), while properly exposed, was (unfortunately) de-centered in acquisition with respect to contemporaneous target imaging. With PSF subtraction this causes wedgeflanking excesses (or deficits) in instrumental brightness, obscuring stellocentric visibilities interior to the WedgeA-1.0 limits achieved, and contributes only "noise" in these smallest stellocentric regions.

$A Q$ data image. For reasons (a)-(d) above, the AQ data images for WedgeA-1.0 and WedgeA-0.6 for HD 207129 combine the PSF-subtracted data as follows:

(1) for WedgeA-1.0: (disk-favored orientation) visits 51-54 are used to an IWA limited by the extent of the wedge,

(2) for WedgeA-1.0: (disk-disfavored orientation) visits 55-58 are used to a larger IWA limited by the PSF saturation distance,

(3) for WedgeA-0.6: (disk-favored orientation) visits 51-54 are used interior to regions unseen with WedgeA-1.0,

(4) for WedgeA-0.6: (disk-disfavored orientation) visits 55-58 are not used due to their inferior image quality.

N.B. With respect to (4), the WedgeA-0.6 AQ images independently confirm the ring structure and morphology seen in the WedgeA-1.0. However, they additionally contribute little - and with noise do not actually improve-knowledge of the very small additional area unsampled with the six-roll WedgeA-1.0 images. Therefore, in this case for quantitative measurements, only the six-roll WedgeA-1.0 combined AQ image is used. 

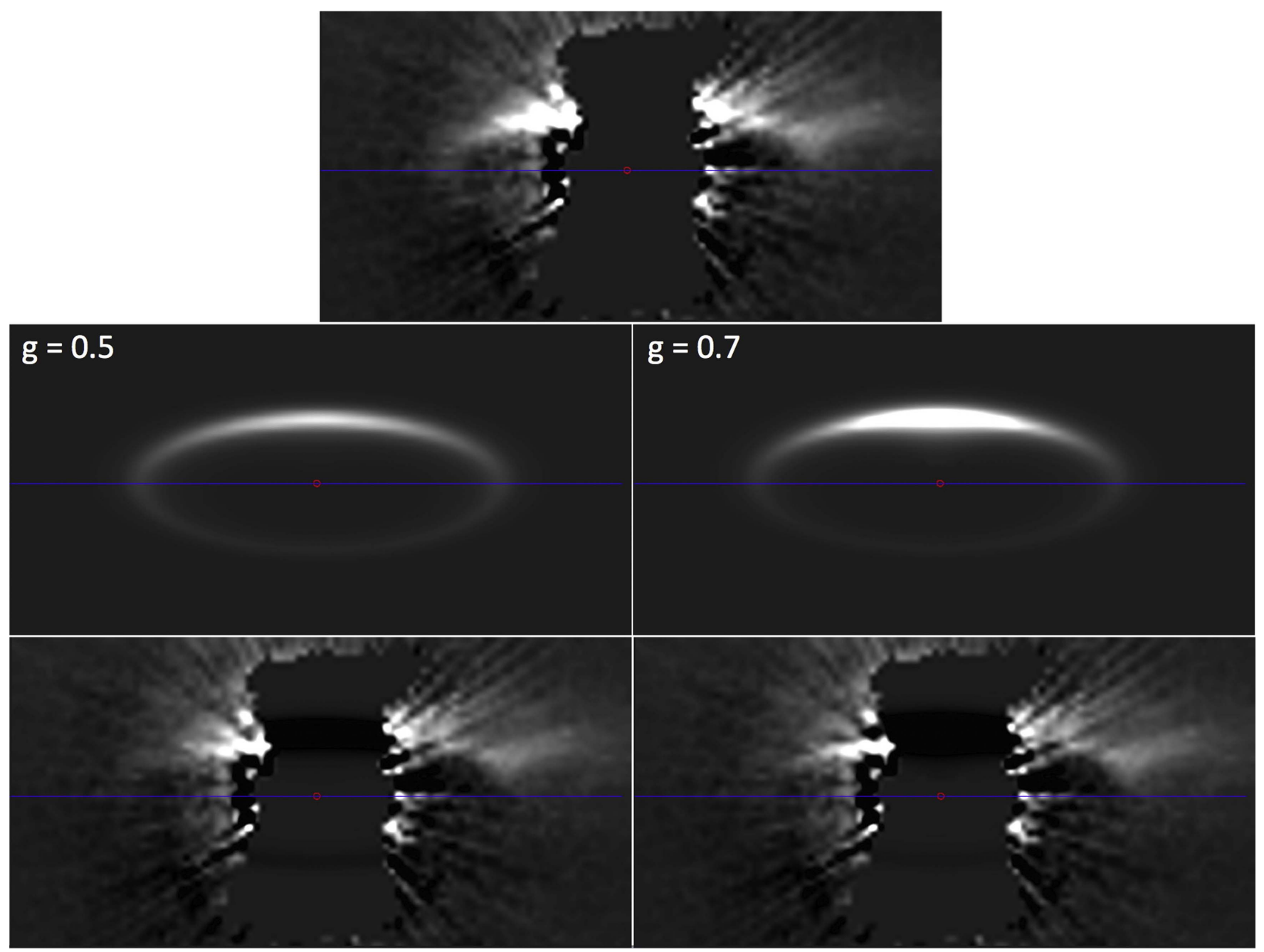

Figure 11. Observed (top), ring models (middle), and observed-minus-model residual (bottom) images of the HD 202917 debris system where significant anisotropic residuals remain (see text). All images have a linear grayscale from -0.2 to +1.0 instrumental counts s ${ }^{-1}$ pixel ${ }^{-1}$, north up, with FOV 5 "! $07 \times 2{ }^{\prime \prime} 53$. Red dots mark the measured location of the occulted star. The horizontal blue lines mark the major axis of the ring.

Roll coverage. The HD 202917 CDS is well revealed in the STIS GO/13786 fully reduced (six-roll combined) AQ image, as shown in Figure 10 with the morphological major axis of its ring-like disk on the image horizontal. Due to constraints on the scheduling of $H S T$, the six field orientations that satisfied other imaging requirements to produce a high-fidelity AQ image could not also provide the complete roll/IWA coverage needed to otherwise fully reveal the debris ring at all CS azimuth angles close to the star. As a result, the debris ring at, and in its opposing sectors closely flanking, its apparent minor axis was not imaged (digitally masked as shown in Figure 10). The remaining unobscured majority of the CDS, however, was imaged with high fidelity.

Principal results.

1. Morphology. Unlike prior ACS and NICMOS reprocessed images (Figure 10, panels (A) and (B)), the STIS image clearly reveals a complex "two-component" CDS morphology with (1) a relatively narrow "half" ring-like disk that is apparently (at least partially) centrally cleared, and (2) a larger and fainter exo-ring with a "fan-like" starlight-scattering structure. The latter is not unlike those seen in some other more highly inclined CDSs, e.g., HD 61005 and HD 32297 (Sch14) wherein, its debris ring is also seen on only one side of the star. Both "components" exhibit significant azimuthal asymmetries.

2. Anisotropic ("front/back") scattering asymmetry. The debris ring itself clearly exhibits a very strong "front/back" asymmetry in scattering phase angle, as suggested from the prior ACS and NICMOS imaging. The SB along the ring is asymmetrically "mirrored" about the major axis of the debris ring. This implicates strongly directionally preferential scattering, with (as a result) the fainter (southern) "half" of the debris ring undetected. The ring itself is seen to "turn around" to the south of both ansae, but only marginally so at its western extremity. The debris ring is undetected from stellocentric celestial P.A. $\approx 120^{\circ}-280^{\circ}$. The exo-ring "fan" of material is, locally, significantly brighter beyond the ring's western ansa than on the diametrically opposed eastern side (see Figure 10 panel (B)). The broad outer scattering "fan" seen only to the north of the star is morphologically skewed, by about $7^{\circ}$ clockwise, with respect to the apparent celestial orientation of the axes of the debris ring (see Figure 10 panel (C)).

3. Model fitting. The best fits to the ring-only model, minimizing the residuals along the visible portion of the debris ring after model subtraction, were found with a HenyeyGreenstein asymmetry parameter $g=0.6 \pm 0.1$ with a 
Table 6

HD 202917 Debris Ring Parameters

\begin{tabular}{|c|c|c|}
\hline $\begin{array}{l}\text { Inclination }(i) \\
\text { P. A. major axis }\end{array}$ & \multicolumn{2}{|c|}{$68.6 \pm 1.5$ from face-on } \\
\hline Inner-edge radius of ring HWHM major axis & $1 "$ " $29 \pm 0$ ". 02 & $55.4 \pm 2.5 \mathrm{au}^{\mathrm{a}}$ \\
\hline Ring width $($ FWHM; $\Delta r)$ & $0 " 13 \pm 0.03$ & $13.16 \pm 1.3 \mathrm{au}^{\mathrm{a}}$ \\
\hline$g$ (HG scattering asymmetry parameter) & & \\
\hline Flux density of debris ring & $182 \pm 36$ counts s ${ }^{-1}$ pixel $^{-1}$ & $83 \pm 17 \mu \mathrm{Jy}$ \\
\hline Total CDS (fan+ring) flux density ${ }^{\mathrm{b}}$ & $\sim 660$ counts s s $^{-1}$ pixel $^{-1}$ & $318 \mu \mathrm{Jy}$ \\
\hline
\end{tabular}

Notes.

${ }^{a}$ Includes uncertainty in stellar parallax from SIMBAD.

b Inner unsampled region obscured by coronagraph wedge and estimated with best-fit model.

correlated uncertainty in scaling the brightness of the model to the observed data by $\pm \sim 20 \%$. In Figure 11 we show these best fits with the model scaled in brightness by $+20 \%$ with $g=+0.5$ and $-20 \%$ with $g=+0.7$ (with the forward scattering direction toward the northern, visible, major axis) in Figure 11. To be clear, we do not attempt to model the exoring "fan," but rather to disambiguate the two "components" of the CDS. Parametric results are summarized in Table 6.

4. Geometry of debris ring. The observed ring geometry has only minor dependences upon $g$ within its estimated uncertainty (see below). In Table 6 we give the best-fit parameters for $g=0.6$ with systematic uncertainties included for solutions with $g$ varied by \pm 0.1 . Fitting the (visible portion of the) debris ring to the best-fit scattering models (Figure 11), we find a semiminor:semimajor axial ratio $\approx 0.182$ with a corresponding inclination $\approx 68^{\circ} .6$ from a face-on viewing geometry for an (assumed) intrinsically circularly symmetric debris ring, with a celestial P.A. of its major axis of $108^{\circ}$ east of north. We find the semimajor axis of the debris ring seen in sky-plane projection $\approx 1$ !" $46(=62.8 \mathrm{au})$ in length as the distance between the SB peaks along the major axis in the best-fit model. The characterizing width of the ring, based upon its full-width to half-intensity maximum from inner edge to outer edge, is $13.2 \mathrm{au}$.

5. Asymmetries and anisotropic scattering. We note two major scattering anisotropies in the HD 207129 CDS. First, the debris ring itself exhibits highly directionally preferential scattering $(g \sim 0.6)$ with its brighter side toward its projected minor axis (Figure 10, panel (A)). The STIS data, unfortunately, did not image the brightest part of the ring. However, the imaged regions along the northern ring-arc appear asymmetrically bright on opposite sides of the minor axis (eastern side brighter). This is perhaps better seen after subtracting the bilaterally symmetric ring models (best fit in the range $0.5<g<0.7$ ), as shown in Figure 10, panel (C), where a bright feature superimposed at the location of the ringarc remains on the eastern side of the unsampled area, but not so on the western side. This suggests that there may be some azimuthally differentiated substructure along the debris ring. Second, scattering by exo-ring material on the western side of the CDS flanking (to the north) of the ring's major axis is significantly brighter than on its otherwise mirror-symmetric eastern side (Figure 10, panel (B)). This suggests a local enhancement in the surface density of scattering particles (through a recent collision or other cause) beyond the bright ring toward celestial P.A. $\sim 300^{\circ}$. This was suggested previously by Soummer et al. (2014) from the PCA-reduced NICMOS image, though the direction of the major axis of the debris ring that we now see is $12^{\circ}$ further clockwise.

6. CDS extent. Exo-ring material of lower surface brightness in the CS "fan" is seen over the same range of scattering phase angles as for the ring itself (suggesting, perhaps, some common physical properties of the material). This material is detected to a maximum stellocentric distance $(+3 \sigma \mathrm{SB}$ above the sky background) of $\sim 5^{\prime \prime}$ toward celestial P.A.s $\approx 90^{\circ}$ and $295^{\circ}$, just to the north of the western ansa of the debris ring where the aforementioned exo-ring bright scattering region is locally seen. This is coincident with the region detected in ACS PSFsubtracted imaging from archival data processed from the MAST. This feature is also likely the origin of the suggestion by Soummer et al. (2014) that the northwest side is significantly brighter (than the SE side), though the significance in the ACS image is not so apparent.

7. Photometry. To estimate the total flux density of the HD 207129 debris ring only, superimposed on its embedding larger CDS structure, we use the best-fit scattering models of the ring with uncertainties in brightness scaling for the range $0.5<g<0.7$. To estimate the total flux density of the full CDS (both the "skirt" and the embedded ring together) we perform area-aperture photometry fully enclosing the CDS and then use the superimposed portion of the ring model coincident with the interior region of the CDS that was unsampled in the STIS image. Both are given in Table 6 , showing that about $2 / 3$ of the total CDS brightness originates in scattering by exo-ring material. With HD 202917 at $V_{\text {mag }}=8.67$, the visible light scattering fraction of the CDS, $F_{\text {disk }} / F_{\text {star }}$, is $\sim 3 \times 10^{-4}$. This is $\sim 40 \times$ "brighter" (in $F_{\text {disk }} / F_{\text {star }}$ ) than both of the $\sim 2.3 \mathrm{Gyr}$ G-star disks discussed in Sections 7.1 and 7.2, and is approximately equal to its IR excess $\left(2.5 \times 10^{-4}\right)$.

\section{DISCUSSION-COMPARATIVE G-STAR DISKOLOGY}

In Section 7 we presented in detail the reduced images and observationally derived metrics characterizing and describing the starlight-scattering debris systems circumscribing three G-type stars: HD 202917, HD 207129, and HD 202628, all with intermediate-inclination ring-like disks. All three systems are very well detected and resolved with STIS 6R-PSFTSC, revealing debris rings that are several times larger than our solar system's Edgeworth-Kuiper Belt, whose inner edge is maintained by Neptune (Liou \& Zook 1999). In the specific case of HD 202628 its stellocentric offset may imply a large planet many times more distant than Neptune is from the Sun. 


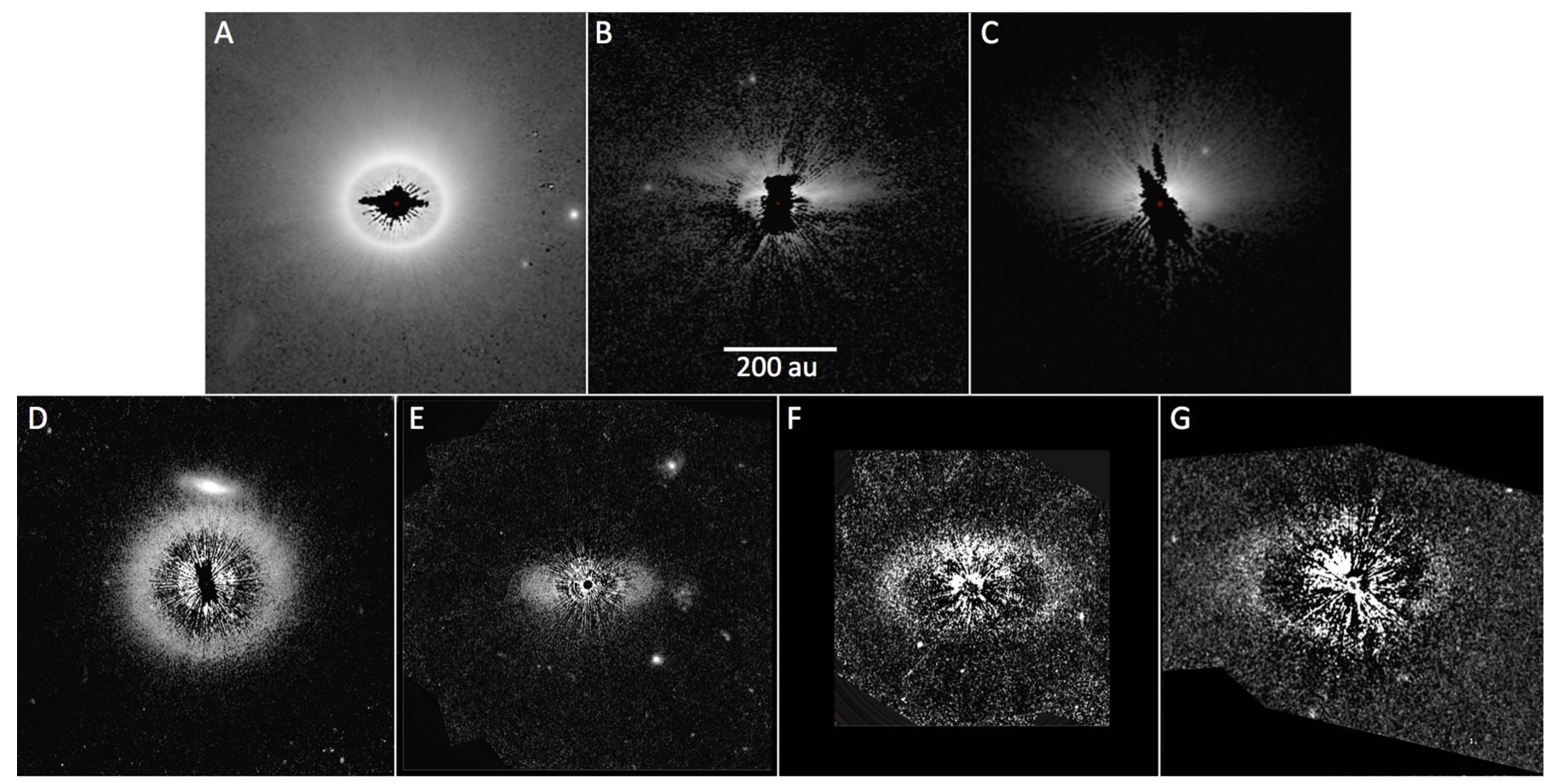

Figure 12. Intermediate-inclination CDSs with well-resolved ring-like components hosted by FGK solar-analog stars as imaged homogeneously with STIS/6RPSFTSC are presented in increasing order (panels (A)-(G)) of stellar age (and apparently correlated with decreasing $F_{\text {disk }} / F_{\text {star }}$; see Figure 13 ). (A) HD 181327 (F6V), (B) HD 202917 (G7V), (C) HD 15745 (F2V), (D) HD 107146 (G2V, with bright background galaxy in the field), (E) HD 92945 (K1V), (F) HD 207129 (G2V), (G) HD 202628 (G5V). A, C, D, and E are from HST program 12228 (Sch14), and B, F, and G from HST program 13786 as discussed in this paper. All are shown at the same physical scale in au (see scale bar applicable to all) with the major axis of their debris rings as seen in sky-plane projection oriented on the image horizontal.

In Figure 12 we present images of these three G-star CDSs on a common physical scale, along with other spatially wellresolved solar-analog (FGK star) CDSs with intermediateinclination ring-like disks that we previously imaged in $H S T$ GO program 12228. Together, these cover an age range from $\sim 23 \mathrm{Myr}$ (panel (A); HD 181327) to $2.3 \mathrm{Gyr}$ (panels (F) and (G)). The latter, HD 207129 and HD 202628, are very mature CDSs of only half the solar age and more massive analogs of our own solar system's circumsolar Edgeworth-Kuiper Belt which has persisted for $\sim 4.6 \mathrm{Gyr}$.

\section{1. $F_{\text {disk }} / F_{\text {star }}$ Brightness over Time}

HD 207129 (G2V) and HD 202628 (G5V), both 2.3 Gyr in age, are the "faintest" solar-analog CDSs yet observed in terms of optical scattering fraction, $F_{\text {disk }} / F_{\text {star }}$, with both $\sim 8 \times 10^{-6}$. Despite their ansal SBs of only $\sim 24.0$ and $24.5 V_{\text {mag }} \operatorname{arcsec}^{-2}$, respectively, they both are readily detectable and mapped with STIS 6R-PSFTSC in part due to the large angular radii of their major axes of $\sim 9$ !' 3 and $\sim 7$ !" 1 . This is, in angular scale, beyond the interior region where PSF subtraction residuals dominate. The optical scattering fractions of these two oldest solar-analog CDSs are in stark contrast to those of the $\sim 23$ Myr HD 181327 (F6V) and $\sim 90 \mathrm{Myr}$ HD 61005 (G8V) with their 200-300 times brighter $F_{\text {disk }} / F_{\text {star }}$.

The well-determined ( $\lesssim 10 \%$ uncertainty) total disk optical scattering fractions of all seven intermediate-inclination FGKstar ring-like CDSs that are shown in Figure 12 are plotted in Figure 13 (left panel) as a function of the estimated ages of their host stars, along with that also of the nearly edge-on HD 61005 (Sch14 and Figure 16). Age estimations and their uncertainties are taken from the literature; see Table 1 of this paper for HD 202917, HD 207129, and HD 202628, Mamajek
\& Bell (2014) for HD 181327, and Table 1 of Sch14 for the other stars ${ }^{19}$ plotted in Figure 13.

Both HD 181327 and HD 61005 have been shown to possess very large, and optically bright, exo-ring scattered-light halos well beyond the peak radii of their thermal IR excess emission (Sch14); for HD 181327 this is posited as arising from a recent massive collision in its birth ring (Stark et al. 2015), and for HD 61005 with small-particle mass loss through a ram-pressure interaction with the local ISM (e.g., Hines et al. 2006). Excluding these two systems with non-steady-state dust production events, the remaining more quiescent CDSs are seen as an ensemble to decay in visible light scattering fractions, $F_{\text {disk }} / F_{\text {star }}$, over time $(t)$ approximated by a $t^{-0.8}$ power law from $\sim 23 \mathrm{Myr}$ to $2.3 \mathrm{Gyr}$. The robustness and more general applicability of this trend, however, should be viewed with due caution given the small sample size and origin. The fit is suggestive of an evolutionary optical dimming timescale for the overall ensemble, but in general may be quite inhomogeneous for individual CDSs that are diverse in stellar host, disk, and environmental characteristics. Additionally, this follow-up sample was culled from sensitivity-limited discovery-mode survey imaging for CDSs with $L_{\mathrm{ir}} / L_{\mathrm{star}} \geqslant 10^{-4}$, and thus could be biased for systems intrinsically fainter in their excess IR emission. The efficacy of the suggested power-law timescale remains to be tested when a larger, and fainter, homogeneous sample of solar-analog debris systems may be available in the future and is necessary to reliably establish a posited evolutionary sequence.

\footnotetext{
${ }^{19}$ For the uncertainty in the age of HD 15745 we adopt a lower value of $\sim 30$ Myr as suggested by Zuckerman \& Song (2004), and for an upper value a canonical age estimation as a likely/probable member of the Castor Moving Group of 200 Myr (Barrado y Navascues 1998).
} 

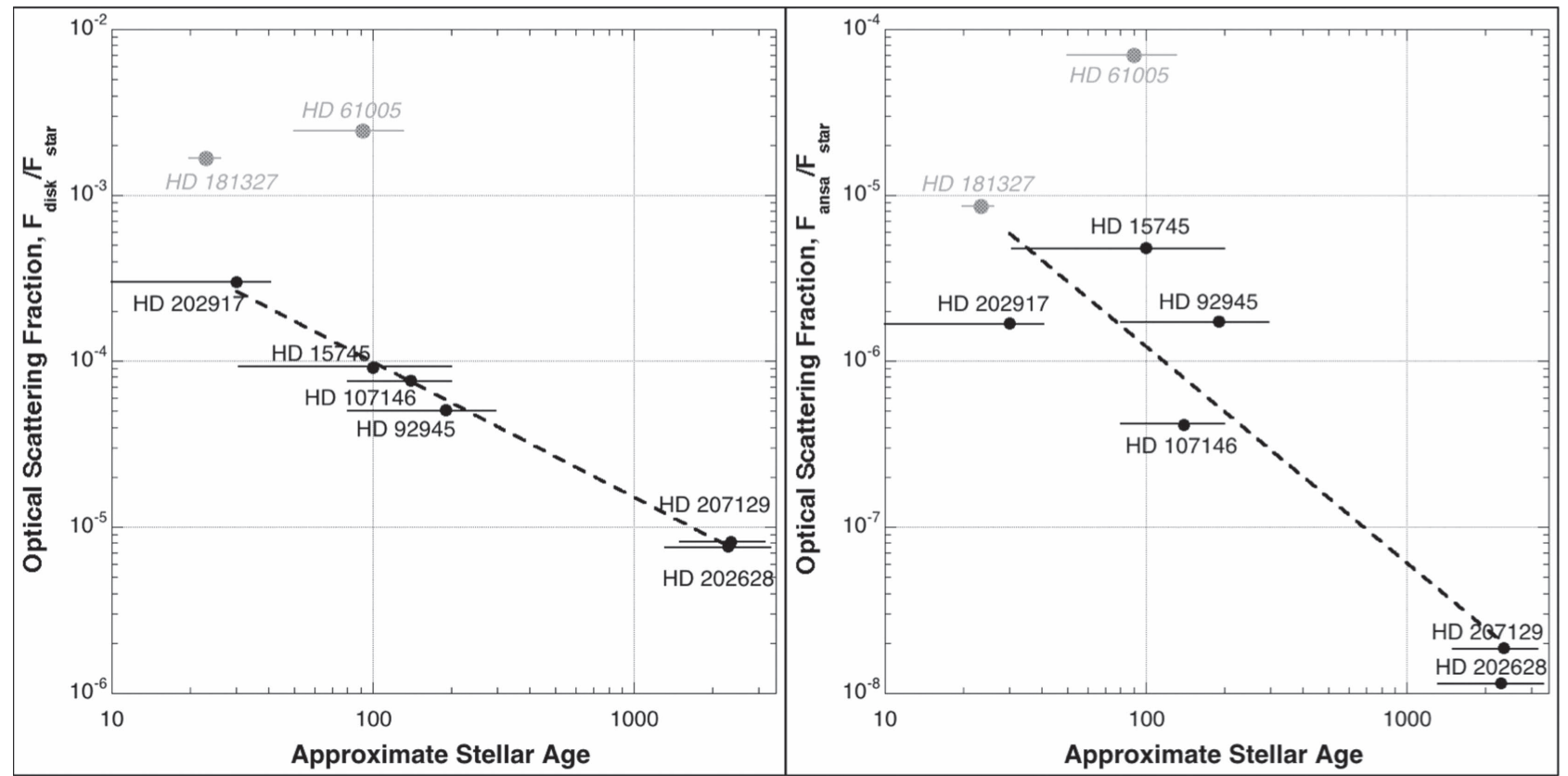

Figure 13. Left: plot of $F_{\text {disk }} / F_{\text {star }}$ as a function of stellar age for the seven intermediate-inclination solar-analog-hosted CDSs shown in Figure 12 , and the nearly edge-on G8V-host outlier HD 61005 (Figure 16). All were measured from nearly identically observed STIS/6R-PSFTSC images. The dashed line is the formal best fit, $t^{-0.81}$, power law excluding outliers HD 181327 and HD 61005 (gray points, see main text). Right: similar spatially localized plot of the 100 au ${ }^{2}$ ansal flux densities of the debris ring compared to the total stellar light and the best-fit (dashed line) power law.

A similar, but somewhat less steep, decay in $24 \mu \mathrm{m}$ IR excess emission, tracing larger particles, was predicted by Gaspar et al. (2013) over the same time for a broader range of host-star spectral types with also less massive disks than our $H S T$ down-selected sample with $L_{\mathrm{ir}} / L_{\mathrm{star}} \geqslant 10^{-4}$. The modeled average disk mass in their population synthesis has not yet reached quasi-steady state and is therefore decaying at a slower rate. However, the more massive members of their population synthesis have, and show, IR decay slopes in agreement with the visible-light observations of the more massive HSTobserved disks. Steeper slopes, more closely matching those seen in our visible-light solar-analog ensemble, are predicted by Gaspar et al. (2013, see their Figure 6) for initially more massive disks.

For each of these spatially well-resolved systems, as an ensemble in Figure 13 (right panel), we also compare the total CDS flux density to that of the flux density in a $100 \mathrm{au}^{2}$ (seen in projection) region of their debris rings at their ansal SB peaks, also ratioed to their host star's flux density ( $\left.F_{\text {ansa }} / F_{\text {star }}\right)$. A somewhat steeper, $\sim t^{-1.3}$, ansal-only power law is seen. Though this is not as well correlated in age for the younger $\left(\lesssim 2 \times 10^{8} \mathrm{Myr}\right)$ systems alone, a similar declining trend is seen with the inclusion of the $(\sim 10 \times)$ oldest systems. The larger dispersion about the trend line of $F_{\text {ansa }} / F_{\text {star }}$ may be due to local anisotropies in the dust density distribution (clumping, deficits, or enhancements from dust production events) in the small $\left(100 \mathrm{au}^{2}\right)$ areas measured in any given system at any given epoch.

Both of the oldest close-solar analog (G-star) CDSs reported in this paper, HD 207129 and HD 202968, have similar IR excesses of $\sim 1 \times 10^{-4}$ (see Table 1). ${ }^{20}$ This is only about an order of magnitude less IR-bright than the $\sim 100 \times$ younger

\footnotetext{
${ }^{20}$ This is three orders of magnitude brighter in $L_{\mathrm{ir}} / L_{\text {star }}$ than our own solar system's circumsolar debris disk.
}

stars in this sample (HD 181327 at $2.5 \times 10^{-3}$ at $\sim 23 \mathrm{Myr}$ and HD 202917 at $1.2 \times 10^{-3}$ at $\sim 30 \mathrm{Myr}$ ) despite their advanced ages. Although no color information is available within the broad STIS spectral passband to assess wavelength dependence (see Section 8.3), the fact that $F_{\text {disk }} / F_{\text {star }}$ for both is an order of magnitude fainter than their IR excesses suggests grains of low optical albedo in these two oldest starlight-scattering CDSs.

\subsection{Morphology and Structure}

Starlight scattered by exoplanetary debris at visible wavelengths traces the small (micron sized) particles in CS environments. When ring-like structures exist, their locations, widths, and inner/outer edge slopes may predictively be able to constrain the dearth/existence, locations, masses, semimajor axes, and eccentricities of co-orbiting planets (Rodigas et al. 2014b). These small particles also react easily to nonplanetary perturbative forces intrinsic to the systems such as stellar corpuscular winds, radiation pressure "pushing" material outward, and Poynting-Robertson drag causing particle orbits to decay inward. CDSs should not, however, be considered as closed or isolated systems. There now exists strong and growing evidence for ISM interactions with the small grain materials at large stellocentric distances in some CDSs manifested with the visual appearance of bow/termination shocks as well as large "skirts" of exo-ring material, for example in HD 61005 (Hines et al. 2007) and HD 32297 (Sch14). The latter may also be the case for HD 202917 and HD 15745 (Figure 14).

\subsubsection{Comparing HD 202917 to HD 15745 and HD 61005}

We compare the morphology of the HD 202917 (Figure 10) CDS with those of HD 15745 (Figure 15) and HD 61005 (Figure 16). All are in the likely middle-age range of 

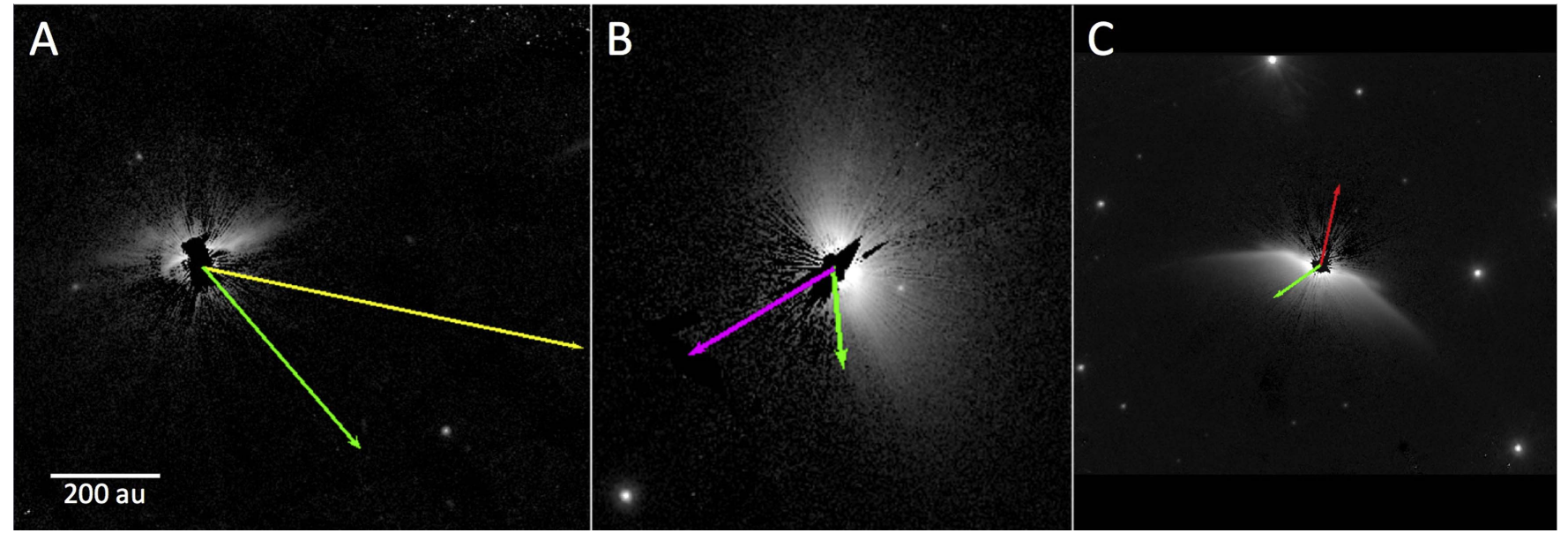

Figure 14. FGK stars hosting CDSs with large skirts or fans of exo-ring materials seen only to one side of the star posited as "blown back" by ISM winds: (A) HD 202917, (B) HD 15745, and (C) HD 61005. Arrows illustrate the proper (tangential) motions of the CDSs with respect to those of the Local Interstellar Cloud (green), and with the other most likely interacting interstellar clouds at their stellar locations. The length of the arrows indicates the stellar/CDS motion through the ISM in 100 years. The most likely other interacting clouds identified from Redfield \& Linsky (2008) are: for HD 202917 the Vel cloud with interaction velocity 7.1 au yr ${ }^{-1}$ (yellow arrow), for HD 15745 the Hyades cloud at $3.1 \mathrm{au} \mathrm{yr}^{-1}$ (purple arrow), and for HD 61005 the "blue" cloud (red arrow) at 1.5 au $\mathrm{yr}^{-1}$. All images are presented north up, east left, at the same physical scale in au along the respective major axes of their rings.
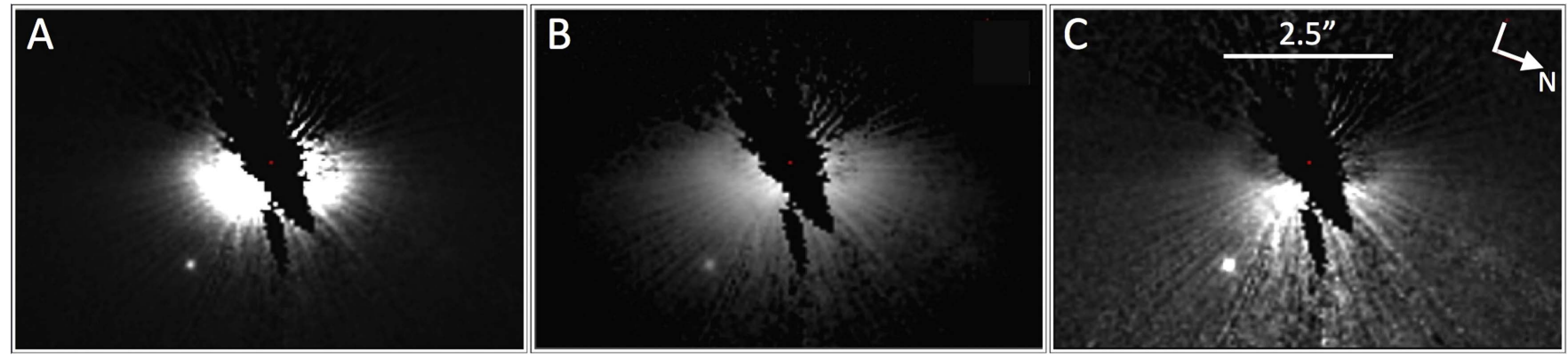

Figure 15. Revealing the starlight-scattering debris ring of HD 15745. Data from HST program 12228 as discussed in Sch14. (A) Linear display from -0.1 to + 3.0 counts s$~^{-1}$ pixel $^{-1}$. (B) $\log _{10}$ display from $[-1.0]$ to $[+1.5]$ dex counts s ${ }^{-1}$ pixel $^{-1}$. (C) Linear display identical to panel (A) after scaling the SB to compensate for the $1 / r^{2}$ falloff in stellar illumination in the plane of the ring. FOV: 7 ! $^{\prime} 6 \times 5$ !" 1 in all three panels.

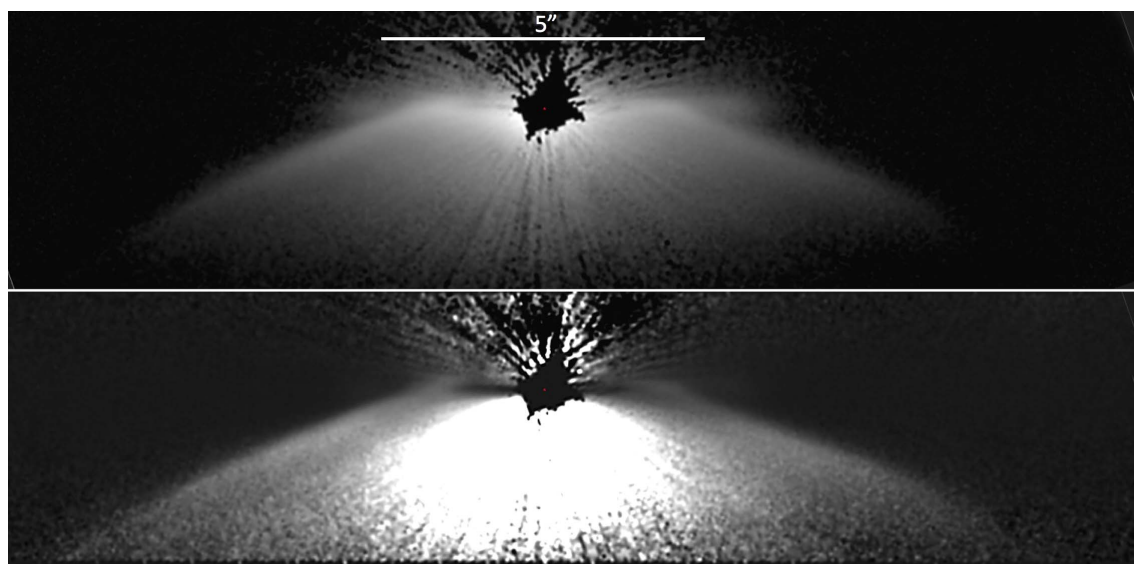

Figure 16. Top: SB image of HD 61005 CDS, as discussed by Sch14, in a $\log _{10}$ display stretch from [-2] to [+1.5] dex counts s ${ }^{-1}$ pixel $^{-1}$. Its debris ring of $\sim 54$ au radius is seen in sky-plane projection along with its exo-ring "skirt" of starlight-scattering material and its leading-edge bow shocks on either side of the ring's minor axis. Bottom: compensating for the $1 / r^{2}$ diminution of stellar light in the (highly inclined) plane of the ring, the central clearing of material in the endo-ring region is readily apparent in this linear display stretch from $[-1]$ to $[+10]$ dex counts s${ }^{-1}$ pixel $^{-1}$. This radial rescaling of the brightness may overenhance the azimuthal brightness asymmetry (anisotropic with scattering phase angle) within the skirt of materials that may be non-coplanar with the debris ring (see footnote 22).

30-90 Myr, with HD 202917 the youngest of the three. All are hosted by FGK stars, with HD 61005 (G8V) a very similar close-solar analog to HD 202917 (G7V). All three bear some significant morphological resemblances with large "skirts" or "fans" of exo-ring scattering material.
In Sch14, the HD 15745 CDS was discussed and presented to best illustrate its extended "fan"-like outer scattering structure (as so originally described from its ACS discovery imaging by Kalas et al. 2007), without emphasis on the debris ring itself. In Figure 15 we show the HD 15745 CDS to better 
illustrate its intermediate-inclination $\left(i \approx 57^{\circ}\right), \quad r=1$ !' 17 (74 au), debris ring within. The ring is a lower-contrast feature, relative to its exo-ring "fan" (or "skirt"), than HD 202917 and HD 61005, and is obscured in a linear representation (Figure 14 panel (A), similar to Figure 13 panel (C) of Sch14). With a logarithmic compression of the dynamic display range, as in panel (B), the debris ring is readily apparent. In panel (C) we scale the SB in the plane of the ring to compensate for the $1 / r^{2}$ dimming of stellocentric illumination, which further enhances the visibility of the ring and of the endo-ring region of the CDS that is relatively devoid of starlight-scattering material. While the region of the ring along its minor axis (in particular flanking its fainter semiminor axis) was unimaged due to HST observing constraints, it is clear that the CDS exhibits highly directionally preferential scattering. This is also the case (more extremely) with HD 202917 at a somewhat similar intermediate $\left(68^{\circ}\right)$ inclination angle.

While the HD $61005 \mathrm{CDS}$ has a highly inclined $\left(\sim 85^{\circ}\right)$ debris ring (unlike those in the intermediate-inclination FGK star sample in Figure 12) it, like HD 202917's, has nonetheless been well detected and resolved in scattered light—at least along its brighter "half" (e.g., Buenzli et al. 2010; Sch14; J. Olofsson et al. 2016, in preparation). Like HD 202917, a highly asymmetric scattering phase function is suggested as being responsible for the non-detection of the fainter "half" of HD 61005's debris ring. Quite differently, however, at its likely intermediate age of $90 \pm 40 \mathrm{Myr}$, the HD 61005 CDS sits well "above" the power-law fit of $F_{\text {disk }} / F_{\text {star }} \sim t^{-0.8}$ to the intermediate-inclination systems plotted in Figure 13.

HD 61005's near edge-on inclination (like also HD 32297's with a more massive $\mathrm{A} 6 \mathrm{~V}^{21}$ host) contributes to the HST detectability of its very large and asymmetric outer scattering structure (see Figure 16, top panel) of small (micron size) starlight-scattering grains. Such asymmetric structures have been posited as arising from an ISM wind blowing back the small grains in the disk halo (Artymowicz \& Clampin 1997). In our viewing geometry this is manifested as a large exo-ring "skirt" of scattering material seen in sky-plane projection. Its endo-ring inner clearing is very apparent when compensating in the ring plane for the $1 / r^{2}$ attenuation of the stellar illumination $^{22}$; see Figure 16, lower panel. The stirring/ launching/ejection of the starlight-scattering exo-ring grains by ISM interaction may contribute to this CDS's notable morphology and to its $F_{\text {disk }} / F_{\text {star }}$ brightness for its age, rather than, e.g., invoking a recent catastrophic collision.

\footnotetext{
${ }^{21}$ Recent suggested reclassification by Rodigas et al. (2014a) with $T_{\text {eff }}=8000 \pm 150 \mathrm{~K}$ and $M=1.65 \pm 0.1 M_{\text {sun }}$.

${ }^{22}$ In this $1 / r^{2}$ presentation, the correction to the in-plane radial illumination that collapses the imaging dynamic also enhances the visibility of azimuthally anisotropic scattering around the exo-ring skirt. This is also the case for the exo-ring fan of HD 15745. However, the exo-ring material may be noncoplanar with the ring, and indeed may possess a more complex threedimensional scattering surface (e.g., an "umbrella geometry") seen in twodimensional projection. Thus, a simplifying assumption of coplanarity may overly enhance this anisotropy with scattering phase angle. While the exo-ring inclination geometry may not be correct as a function of stellocentric distance, the cause of the enhancement is probably a real effect. The scattering phase angle directly "below" the star on the system's minor axis $(\theta=0, z$ increasing downward in cylindrical coordinates) should be the minimum scattering angle as a function of $z$, precisely where an HG scattering phase function is peaking. Hence, a peak in SB directly below the star is expected for a "fixed" $z$ (ignoring inclination). Detailed modeling beyond the scope of this paper (D. C. Hines et al. 2016, in preparation) can constrain the inclinations of the exo-ring lightscattering material.
}

The HD 202917 CDS, though less highly inclined to our line of sight than the HD $61005 \mathrm{CDS}$, bears morphological resemblance (at lower $\mathrm{S} / \mathrm{N}$ ) in its SB image (compare Figure 10 panel (C) to Figure 16 top). In both of these systems only the brighter "half" of the debris ring with a very large front-to-back scattering asymmetry is detected. Both, along with HD 15745 , also possess large skirts or fans of exo-ring scattering material seen on the brighter side only of the CDSs. HD 202917 differs, however, from both HD 61005 and HD 15745 in that it is significantly asymmetrically brighter on one side (the east) of the ring's minor axis at and beyond the ring itself. The morphology of such a structure may be difficult to reproduce through an ISM interaction alone, and may be indicative of a recent dust enhancement event through a catastrophic collision or other dust production event. Kalas et al. (2007) disfavored ISM blowback as a cause of the HD 202917 fan, noting the star's tangential motion and location well within the local bubble. However, a plausibly analogous bow shock seen in IR observations of $\delta$ Vel (Gaspar et al. 2008), also in the local bubble with similar relative motion to the ISM, may suggest otherwise and is indicative of the fact that cloud patches of higher density exist in the local bubble. Reproducing the morphology of the HD 202917 CDS is a challenge to models of the structural evolution of disks inclusive of forces both intrinsic and extrinsic to the system (beyond the scope of this current paper).

\subsubsection{Intercomparing the Oldsters-HD 207129 and $H D 202628$}

We also intercompare the two, approximately equally oldest ( 2.3 Gyr), G-star CDSs yet imaged, HD 207129 (G2V) and HD 202628 (G5V). Both share similar ring-like morphologies, are roughly comparable in size $(a=149$ au and $175 \mathrm{au}$, respectively), and have very similar optical scattering fractions $\left(\sim 8 \times 10^{-6}\right)$. The HD 207917 CDS exhibits a high degree of biaxial morphological symmetry, with its azimuthally varying SB well fit with a Henyey-Greenstein scattering model with $g \sim+0.25$. HD 202628 appears axially mirror-symmetric about its apparent major axis (only) with only a small asymmetry in scattering phase $(g \sim+0.1)$. The HD 207129 debris ring appears centered on its stellar host, while HD 202628 is stellocentrically offset. No exo-ring material was detected exterior to the soft outer edge of the HD 207129 debris ring, while a diffuse "cloud" of low-SB exo-ring scattering material appears extending outward from the stellocentrically more distant ansa only of the HD 202628 debris ring. This (so far) unique CDS asymmetry may (or may not) be associated with the stellocentric offset of the debris ring itself. The combination of its stellocentric offset and its diffuse exo-ansal cloud give HD 202628 its distinctively unique "teardrop" morphology. Additionally, after subtracting a SB model of the ring-like component of the HD 202628 CDS, a number of intra-ring "clumps" are revealed. While some (or even all) of these may arise from background contamination, their spatial distribution preferentially superimposed upon the location of the debris ring itself, and absent elsewhere, suggests that at least some of these are likely real from local enhancements in dust density and not image artifacts. Reproducing these features in the HD 202628 CDS that do not appear with HD 207129 may present a challenge to future models of these two systems with very similar stellar hosts. 


\subsection{Fundamental Photometric Data for Modeling Material Properties}

Both filter-band color diagnostics and directionally preferential scattering derived from spatially resolved imaging photometry can inform or constrain the physical properties of the starlight-scattering materials (e.g., Debes et al. 2008a, 2008b). The former, however, cannot be obtained with STIS coronagraphic observations alone. However, the flux density and SB measures in the STIS 50CCD spectral band that we report in Tables 4-6 may be combined with future data to be obtained with continuing improvements to ground-based highcontrast imaging systems in the near IR, and with the advent of James Webb Space Telescope/nirCAM coronagraphy. Separately, photometrically determined scattering efficiencies as a function of the phase angle of stellocentric illumination with spatially resolved imaging, even in a single spectral band, parameterized by $g$, can provide a useful constraint in ascertaining the physical properties of materials in some CDSs. Unlike face-on or edge-on CDSs, the intermediate-inclination CDSs we focus on in this paper simultaneously provide an explorable range of circum-azimuthal scattering phase angles (lacking in face-on disks) that (with sufficient resolution and image contrast) are spatially resolvable with significance and without self-obscuration of debris structures (in edge-on disks).

In fitting the STIS 50CCD band SB distributions of the ringlike components of the intermediate-inclination CDSs of HD 202917, HD 207129, and HD 202628, we assumed a simple, single characterizing $\mathrm{HG}$ asymmetry parameter, $g$; see Section 6. More completely descriptive scattering models, e.g., those considering optical properties dependent upon a diversity in material composition, sizes, and mixes, are beyond the intended scope of this paper. Also see, e.g., Stark et al. (2014) for a discussion of the necessity for alternative or more complex formulations for some CDSs as explored in part for HD 181327. For the three G-star CDS rings we have observed, over the range of explorable scattering phase angles ${ }^{23}$, we found a range from nearly isotropic scattering (HD 202628, $g \approx 0.1$ ) to mildly asymmetric scattering (HD 207129, $g \approx 0.25)$ in the case of the two oldest CDSs, to strongly asymmetric scattering (HD 202916, $g \approx 0.6$ ) such that only the brighter side of the debris ring (centered on $\varphi=0^{\circ}$ ) was detectable.

\section{SUMMARY AND CONCLUSIONS}

(1) We have used STIS six-roll PSF-template-subtracted coronagraphy to explore the morphological, photometric, and astrometric properties of the endo-, intra-, and exo-ring environments of three CDSs with G-star hosts. STIS 6RPSFTSC provides unique access to large ( $>$ a few arcseconds) and spatially diffuse, CDS substructures at low SB and high contrast beyond the outer working limits of current state-of-theart high-contrast AO-augmented imaging systems (e.g., GPI high-contrast half-angle field limit $=1$ ". 7 ). Imaging data were obtained in total light at visible wavelengths and without reliance on flux non-conservative reduction methods (such as ADI commonly used on the ground for contrast-augmentation) that otherwise often "self-subtract" low-contrast diffuse structures, increasing uncertainties in morphological and photometric interpretation.

\footnotetext{
23 HD 202628: $\varphi= \pm\left(35^{\circ} .8\right.$ to 144.2$)$, HD 207129: $\varphi= \pm\left(32.1\right.$ to $\left.147^{\circ} .9\right)$, HD 202917: $\varphi= \pm\left(21.4\right.$ to $\left.158^{\circ} .6\right)$.
}

(2) For HD 207129, one of the two comparably old $(\approx 2.3 \mathrm{Gyr})$ stars in our sample, we find a biaxially symmetric ring-like debris disk exhibiting HG-like scattering with $g=0.25 \pm 0.05$ (differing from discovery imaging of Krist et al. 2010). The debris ring peaks in radial SB at a stellocentric distance of $\approx 150 \mathrm{au}$, with a characteristic width of $\approx 73 \mathrm{au}$, and may be marginally brighter at its NW ansa than at its SE ansa. Interior to the ring, with an inner (FWHM) edge radius of $\approx 116 \mathrm{au}$, we find no additional evidence of starlight-scattering dust to a limiting sensitivity in surface brightness of $\sim 23 V_{\text {mag }} \operatorname{arcsec}^{-2}$ at $r=5^{\prime \prime}$ (80 au) from the star, nor beyond its outer periphery to a limit of $\approx 25.8 \pm 0.3 V_{\text {mag }} \operatorname{arcsec}^{-2}$. The total optical-light scattering fraction of the disk compared to the star, $F_{\text {disk }} / F_{\text {star }}=(8.2 \pm 0.8) \times 10^{-6}$, is about an order of magnitude (a factor of 13) less than its thermal IR excess, suggestive, perhaps, of low-albedo grains. We confirm through two-epoch non-common proper motions that three point-like objects seen initially in the discovery imaging of Krist et al. (2010) are not physically associated with the CDS, so if the ring is sculpted by one or more co-orbiting planets they remain undetected.

(3) For HD 202628, of comparable age to HD 207129, we find its debris ring with $a \approx 175$ au to be more azimuthally isotropically scattering with $g=0.1 \pm 0.1$ and confined to a sky-plane-projected elliptical annulus from $~ 135$ to 218 au. Its CDS morphology and structure are more complex with also (a) a low-SB cloud of scattering material superimposed on and exterior to the debris ring SE (only) ansa, (b) a stellocentric offset of the ring itself (initially suggested by Krist et al. (2012), confirmed here, but found in a somewhat different amount), and (c) a suggestion of spatially resolved debris "clumps" along the ring, most notably to the SW of the star. Together, (a)-(c) may implicate a much more dynamic environment perturbing the dust distribution, which is absent from HD 202628. From (c) an $r^{-2}$ SB scaling would be expected with differing ansal distance, but is apparently modified by the presence of (a) at the SE ansa, a further indication of a more asymmetrically complex overall dust density distribution. Qualitatively, (a) and (b) give the system when imaged at high sensitivity to large stellocentic distance (comparable to that of HD 207129) a distinctly asymmetrical "teardrop" morphology seen uniquely in HD 202628. Similar to HD 207129, though, HD 202628's $F_{\text {disk }} / F_{\text {star }} \approx 7.7 \times 10^{-6}$ is also an order of magnitude (a factor of 18) less than its thermal IR excess.

(4) HD 202917 is the youngest ( $30 \mathrm{Myr}$ ), by about two orders of magnitude, in age of the three close-solar analog CDSs in this sample. Its 13 au wide debris ring with $a=$ $63 \mathrm{au}$ is seen to be highly anisotropically scattering azimuthally. With $g=0.6 \pm 0.1$, only the much brighter "half" of its $68^{\circ} .6$ inclined debris ring, imaged through a range of scattering phase angle of $\pm 21^{\circ}-159^{\circ}$, is detectable. HD 202917 possesses a highly complex and asymmetric exo-ring scattering structure, seen only beyond the brighter side of the ring itself and also with a strong asymmetry in exo-ansal brightness. This "fan-like" exo-ring structure bears some morphological resemblance to the exo-ring "skirt" seen (at higher S/N) in the HD 61005 CDS, suggestive, perhaps, of a common origin or cause, though the anisotropy in ansal brightness may betray a recent conflating dust production event. Unlike HD 207129 and HD 202628, HD 202917's much brighter $F_{\text {disk }} / F_{\text {star }} \approx 3 \times 10^{-4}$ is nearly identical to its IR 
excess emission, as may be anticipated for younger CDSs; e.g., see Figure 18 of Sch14.

(5) Combining the above three solar-analog CDSs with others we had previously observed as a small but homogeneous sample is cautiously suggestive of an diminution in disk brightness $F_{\text {disk }} / F_{\text {star }}$ over time $(t)$ with a power-law slope approximately $t^{-0.8}$, although sporadic dust production/ enhancement events in individual systems may bias this suggested trend (as exemplified by HD 181327 and HD 61005).

(6) As an ensemble inclusive now of HD 15745, when imaged with sufficient depth and sensitivity as enabled with STIS 6R-PSFTSC, the number of CDSs exhibiting very large exo-ring scattering structures that are massive analogs to the outer regions of our solar system's Kuiper Belt is growing, and such CDSs may be common. Such structures imaged in visible light uniquely trace the small grains that may be in the process of expulsion from these systems and/or interacting with their local interstellar environments (e.g., Figure 14). Their presence informs that ongoing and future holistic models of CDS structure and evolution cannot treat debris systems in isolation.

Based on observations made with the NASA/ESA Hubble Space Telescope, obtained at the Space Telescope Science Institute (STScI), which is operated by the Association of Universities for Research in Astronomy, Inc., under NASA contract NAS 5-26555. These observations are associated with programs \#13786 and 12228. Support for program \#13786 was provided by NASA through a grant from STScI. J. Carson acknowledges support from the South Carolina Space Grant Consortium.

\section{REFERENCES}

Apai, D., Schneider, G., \& Grady, C. A. 2015, ApJ, 800, 136

Artymowicz, P., \& Clampin, M. 1997, ApJ, 490, 863

Barrado y Navascues, D. 1998, A\&A, 339, 831
Beichman, C. A., Bryden, G., Rieke, G. H., et al. 2005, ApJ, 622, 1160 Boccaletti, A., Thalmann, C., Lagrange, C., et al. 2015, Natur, 526, 230 Buenzli, E., Thalmann, C., \& Vigan, A. 2013, A\&A, 524, 1 Choquet, É., Perrin, M. D., Chen, C., et al. 2016, ApJ, 817, 2

Debes, J., Jang-Condell, H., \& Schneider, G. 2016, ApJ, 819, 1

Debes, J., Weinberger, A. J., \& Schneider, G. 2008a, ApJL, 682, L145

Debes, J., Weinberger, A. J., \& Schneider, G. 2008b, ApJL, 673, L191

Gáspár, A., Rieke, G. H., \& Balog, Z. 2013, ApJ, 768, 25

Gaspar, A., Su, K., \& Rieke, G. 2008, ApJ, 672, 974

Hedman, M. M., \& Stark, C. C. 2015, ApJ, 811, 67

Henyey, L. G., \& Greenstein, J. L. 1941, ApJ, 93, 70

Hines, D. C., Schneider, G., Hollenbach, D., et al. 2007, ApJL, 671, L165

Jordin de Muizon, M., Laurejis, R. J., Dominik, C., et al. 1999, A\&A, 350, 975

Kalas, P., Duchene, G., Fitzgerald, M. P., et al. 2007, ApJL, 671, L161

Koerner, D. W., Kim, S., Trilling, D. E., et al. 2010, ApJL, 710, L26

Konishi, M., Grady, C. A., Schneider, G., et al. 2016, ApJL, 818, 23

Krist, J. 2007, in Spirit of Bernard Lyot: The Direct Detection of Planets and Circumstellar Disks in the 21st Century, ed. P. Kalas (Berkeley, CA: Univ. California) http://w.astro.berkeley.edu/ kalas/lyot2007/Presentations/ Krist_John.pdf

Krist, J., Stapelfeldt, K. R., Bryden, G., et al. 2010, AJ, 140, 1051

Krist, J., Stapelfeldt, K. R., Bryden, G., et al. 2012, AJ, 144, 45

Liou, J.-C., \& Zook, H. A. 1999, AJ, 118, 580

Lohne, T., Eiroa, C., Augereau, J.-C., et al. 2012, A\&A, 537, A110

Mamajek, E. E., \& Bell, C. P. M. 2014, MNRAS, 445, 2169

Mamajek, E. E., \& Hillenbrand, L. A. 2008, ApJ, 687, 1264

Marshall, J. P., Lohne, T., Montesinos, B., et al. 2011, A\&A, 529, A117

Moór, A., Ábrahám, P., Derekas, A., et al. 2006, ApJ, 644, 525

Redfield, S., \& Linsky, J. L. 2008, ApJ, 673, 283

Rodigas, T. J., Debes, J. H., Hinz, P. M., et al. 2014a, ApJ, 783, 21

Rodigas, T. J., Malhorta, R., \& Hinz, P. M. 2014b, ApJ, 780, 65

Schneider, G., Grady, C. A., Hines, D. C., et al. 2014, AJ, 148, 59

Schneider, G., Silverstone, M., Stobie, E., et al. 2010, in 2010 Space Telescope Science Institute Calibration Workshop-Hubble after SM4, ed. S. Deustra \& C. Oliveira (Baltimore, MD: STScI), 15

Schneider, G., Silverstone, M. D., \& Hines, D. C. 2006, ApJ, 650, 414

Schneider, G., Smith, B. A., Becklin, E. E., et al. 1999, ApJ, 513, 127

Silverstone, M. D. 2000, PhD thesis, UCLA

Soderblom, D. R. 1985, PASP, 97, 54

Soummer, R., Perrin, M. D., Pueyo, L., et al. 2014, ApJ, 786, 23L

Stark, C., Kuchner, M. J., \& Lincowski, A. 2015, ApJ, 801, 128

Stark, C., Schneider, G., Weinberger, A. J., et al. 2014, ApJ, 789, 58

Walker, H. J., \& Wolsterncroft, R. D. 1988, PASP, 100, 1509

Zuckerman, B., \& Song, I. 2004, ApJ, 603, 738 